\title{
SUMMARY OF WATER DATA FOR THE JANZEN RECHARGE INVESTIGATION, SCOTT COUNTY, KANSAS, 1980-86
}

By Lloyd E. Stullken

U.S. GEOLOGICAL SURVEY

Open-File Report 88-83

Prepared in cooperation with the WESTERN KANSAS GROUNDWATER MANAGEMENT DISTRICT NO. 1 


\section{DEPARTMENT OF THE INTERIOR \\ DONALD PAUL HODEL, Secretary \\ U.S. GEOLOGICAL SURVEY}

Dallas L. Peck, Director

For additional information

write to:

District Chief

U.S. Geological Survey

1950 Constant Ave - Campus West

Lawrence, Kansas 66046

\section{Copies of this report can be purchased}

from:

U.S. Geological Survey

Books and Open-File Reports

Federal Center, Building 810

Box 25425

Denver, Colorado 80225 


\section{CONTENTS}

Page

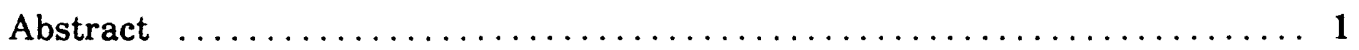

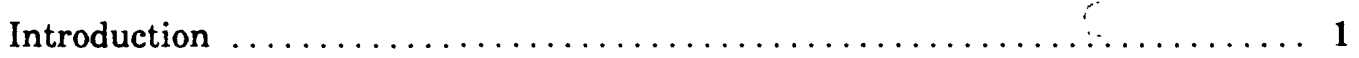

Description of dam and reservoir $\ldots \ldots \ldots \ldots \ldots \ldots \ldots \ldots \ldots \ldots \ldots$

Reservoir water levels $\ldots \ldots \ldots \ldots \ldots \ldots \ldots \ldots \ldots \ldots \ldots \ldots \ldots \ldots \ldots$

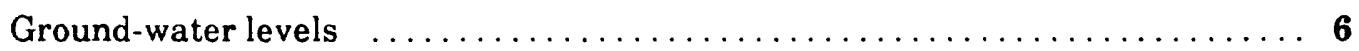

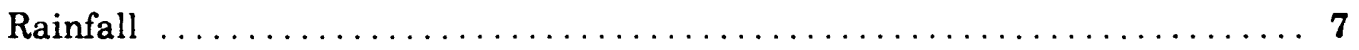

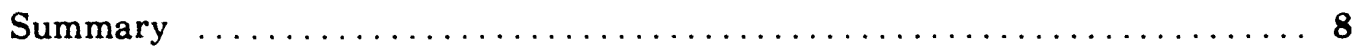

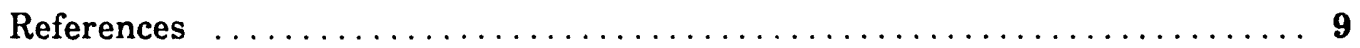

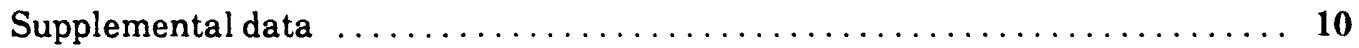

$\begin{array}{ll}\text { Figure } & \text { Page }\end{array}$

1. Map showing location of report area and damsite $\ldots \ldots \ldots \ldots \ldots \ldots 2$

2. Map showing location of data-collection instrumentation at damsite $\ldots \ldots \quad 3$

Figures 1-6. Graph showing:

3. Reservoir gage heights during periods of water storage $\ldots \ldots \ldots \ldots \ldots$ 5

4. Water levels in observation well 1, April 1 to May $31,1984 \ldots \ldots \ldots \ldots 6$

5. Five-day water levels in observation well 1 and recorded daily rainfall for period of record $\ldots \ldots \ldots \ldots \ldots \ldots \ldots \ldots \ldots \ldots \ldots$

6. Water levels in observation wells at Janzen damsite $\ldots \ldots \ldots \ldots \ldots \quad 8$

Table . Page

1. Reservoir storage capacity $\ldots \ldots \ldots \ldots \ldots \ldots \ldots \ldots \ldots \ldots \ldots \ldots$

2. Hourly reservoir gage heights, April 2-15, 1984, April 30 to May 9,

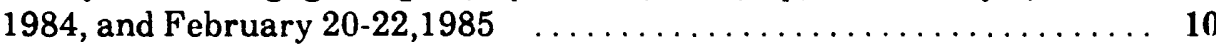


Table Page

3. Hourly recorded depth to water in observation well 1, April 1 to May 31, $1984 \ldots 16$

4. Five-day recorded depths to water in observation well $1 \ldots \ldots \ldots \ldots \ldots \ldots \ldots$

5. Measurement of depths to water in observation wells 1, 2, and 3 made during

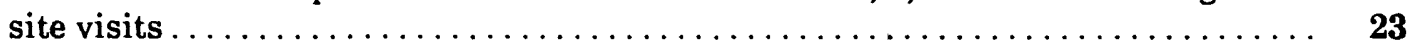

6. Summary of daily rainfall for 1983 , in inches $\ldots \ldots \ldots \ldots \ldots \ldots \ldots \ldots \ldots$

7. Summary of daily rainfall for 1984 , in inches $\ldots \ldots \ldots \ldots \ldots \ldots \ldots \ldots \ldots$

8. Summary of daily rainfall for 1985 , in inches $\ldots \ldots \ldots \ldots \ldots \ldots \ldots \ldots \ldots \ldots$

9. Summary of daily rainfall for 1986 , in inches $\ldots \ldots \ldots \ldots \ldots \ldots \ldots \ldots \ldots \ldots \ldots$

10. Construction data for observation wells. . . . . .

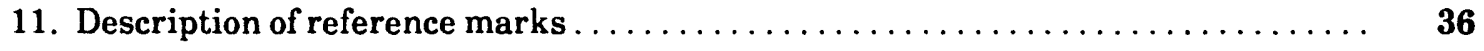

12. Summary of reference levels $\ldots \ldots \ldots \ldots \ldots \ldots \ldots \ldots \ldots \ldots \ldots \ldots \ldots \ldots \ldots, \quad 38$

\section{CONVERSION TABLE}

For those readers interested in metric units, the inch-pound units used in this report can be converted to the International System of Unit (SI) using the following factors:

\begin{tabular}{|c|c|c|}
\hline $\begin{array}{l}\text { Multiply } \\
\text { inch-pound unit }\end{array}$ & By & $\begin{array}{l}\text { To obtain } \\
\text { SI unit }\end{array}$ \\
\hline inch & 25.4 & millimeter \\
\hline foot & 0.3048 & meter \\
\hline mile & 1.609 & kilometer \\
\hline square mile $\left(\mathrm{mi}^{2}\right)$ & 2.590 & square kilometer \\
\hline acre & 4,047 & square meter \\
\hline acre-foot & 1,233 & cubic meter \\
\hline $\begin{array}{l}\text { cubic foot per second } \\
\left(\mathrm{ft}^{3} / \mathrm{s}\right)\end{array}$ & 0.02832 & cubic meter per second \\
\hline foot per day & 0.3048 & meter per day \\
\hline
\end{tabular}

Sea level: In this report "sea level" refers to the National Geodetic Vertical Datum of 1929 (NGVD of 1929)--a geodetic datum derived from a general adjustment of the first-order level nets of both the United States and Canada, formerly called "Mean Sea Level of 1929." 


\title{
SUMMARY OF WATER DATA FOR THE JANZEN RECHARGE INVESTIGATION, SCOTT COUNTY, KANSAS, 1980-86
}

\author{
By Lloyd E. Stullken
}

\begin{abstract}
The U.S. Geological Survey, in cooperation with the Western Kansas Groundwater Management District No. 1, established data-collection instrumentation around an earthen dam in Scott County, west-central Kansas. The dam was located on an ephemeral drainage to monitor water storage behind the dam, precipitation, and ground-water levels in the vicinity. This report briefly describes the site and summarizes the data collected at that site from May 1980 through September 1986 , when data collection was discontinued.

The dam is capable of impounding 300 acre-feet of water at the level of the emergency spillway, draining to 24 acre-feet of water at the level of the principal spillway. The reservoir behind the dam receives surface-water runoff from a drainage area of about 8,000 acres. Water storage behind the dam occurred three times during the period of data collection, but water never reached the level of the principal spillway.
\end{abstract}

\section{INTRODUCTION}

Artificial recharge to an aquifer is one way to replenish the diminishing supply of ground water in western Kansas. In an area where surface water is limited, investigations of aquifer recharge may use artificial storage to catch and provide a water source. In the investigation described in this report, Western Kansas Groundwater Management District No. 1 sponsored construction of an earthen dam on a tributary to Ladder Creek in the NW1/4 of section 11, T. $17 \mathrm{~S} ., \mathrm{R} .34 \mathrm{~W}$., northeastern Scott County (fig. 1). Construction was under the supervision and to the specifications of the U.S. Department of Agriculture, Soil Conservation Service. Included in the investigation were hree ground-water observation wells around the perimeter of the water-storage area. A map of the study site is shown in figure 2 .

The U.S. Geological Survey, in cooperation with the Western Kansas Groundwater Management District No. 1, completed data-collection instrumentation around the damsite in August 1982, to monitor water storage behind the dam, precipitation, and ground-water levels in the vicinity. This report briefly describes the site and summarizes the data collected at the site through September 1986, when data collection was discontinued. 


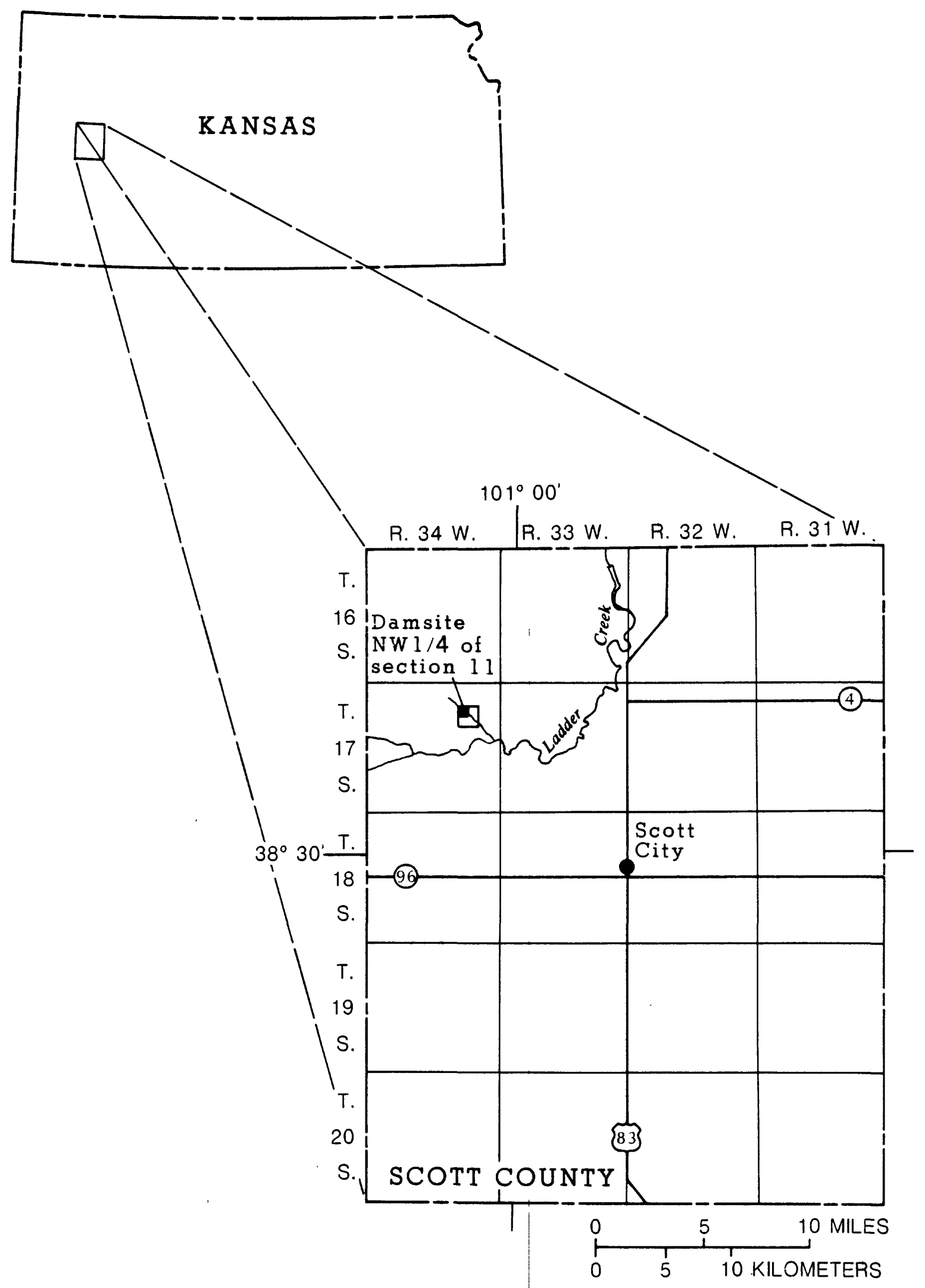

Figure 1. Location of report area and damsite. 


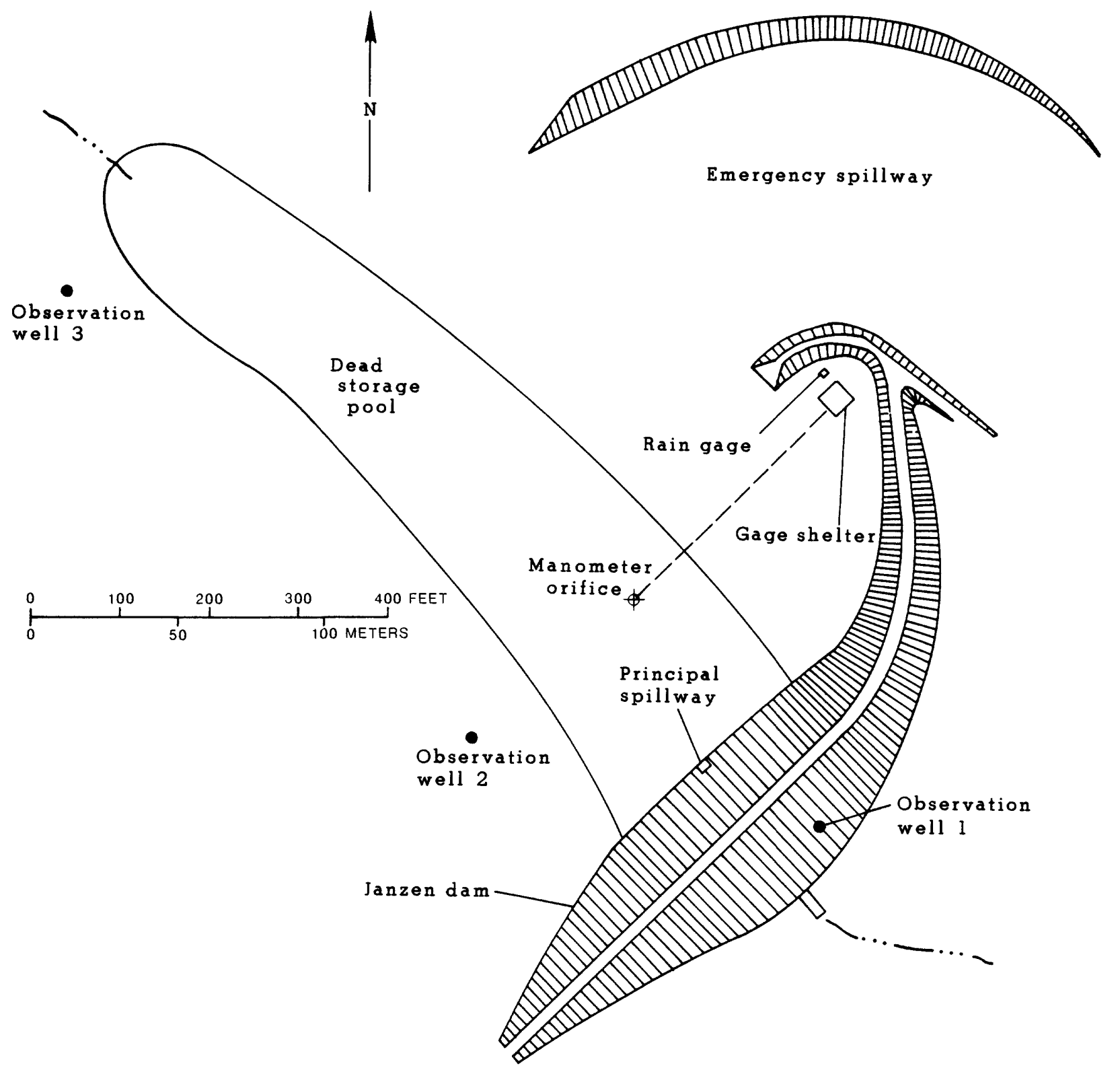

Figure 2. Location of data-collection instrumentation at damsite.

\section{DESCRIPTION OF DAM AND RESERVOIR}

The dam at Janzen is a compacted earthfill capable of storing about 24 acre-feet of water below the inlet to the principal spillway (36-inch corrugated metal pipe). It was constructed during the winter and spring of 1981-82. At the crest of the emergency spillway the structure impounds about 300 acre-feet of water. At that level the discharge through the principal spillway pipe would be about $113 \mathrm{ft} 3 / \mathrm{s}$ (table 1). At the top of the dam, about 480 acre-feet of water would be impounded, and outflow would be about $5,000 \mathrm{ft} 3 / \mathrm{s}$. 
Table 1. Reservoir storage capacity

[Modified from Wallace, 1981]

\begin{tabular}{|c|c|c|c|c|c|}
\hline \multicolumn{3}{|c|}{ Elevations, in feet 1} & \multirow[b]{2}{*}{$\begin{array}{c}\text { Storage } \\
\text { (acre- } \\
\text { feet) } \\
\text { second) } 2\end{array}$} & \multirow[b]{2}{*}{$\begin{array}{l}\text { Outflow } \\
\text { (cubic } \\
\text { feet per } \\
\text { second) }\end{array}$} & \multirow[b]{2}{*}{ Remarks } \\
\hline $\begin{array}{l}\text { Above } \\
\text { gage } \\
\text { datum }\end{array}$ & $\begin{array}{l}\text { Above sea } \\
\text { level }\end{array}$ & $\begin{array}{l}\text { Above } \\
\text { dam } \\
\text { datum }\end{array}$ & & & \\
\hline 4.1 & $3,060.3$ & 26.5 & 0.0 & -- & \\
\hline 7.6 & $3,063.8$ & 30.01 & .08 & -. & \\
\hline 11.6 & $3,067.8$ & 34.0 & 6.36 & -- & \\
\hline 15.6 & $3,071.8$ & 38.0 & 18.92 & -. & \\
\hline 16.73 & $3,072.9$ & 39.1 & 23.97 & 20.0 & $\begin{array}{l}\text { Crest of principal } \\
\text { spillway. }\end{array}$ \\
\hline 17.2 & $3,073.4$ & 39.6 & -- & 2.6 & \\
\hline 17.7 & $3,073.9$ & 40.1 & -. & 5.2 & \\
\hline 18.2 & $3,074.4$ & 40.6 & -- & 8.6 & \\
\hline 18.7 & $3,074.9$ & 41.1 & -- & 16.9 & \\
\hline 19.2 & $3,075.4$ & 41.6 & -. & 25.3 & \\
\hline 19.6 & $3,075.8$ & 42.0 & 40.84 & -. & \\
\hline 19.7 & $3,075.9$ & 42.1 & -- & 33.7 & \\
\hline 20.0 & $3,076.2$ & 42.4 & -- & 42.1 & \\
\hline 22.1 & $3,078.3$ & 44.5 & -- & 81.5 & \\
\hline 23.6 & $3,079.8$ & 46.3 & 73.92 & $\cdots$ & \\
\hline 25.25 & $3,081.4$ & 47.62 & -- & 89.7 & \\
\hline 27.6 & $3,083.8$ & 50.0 & 123.02 & -- & \\
\hline 29.19 & $3,085.4$ & 51.56 & -- & 99.1 & \\
\hline 31.6 & $3,087.8$ & 54.0 & 198.28 & -- & \\
\hline 33.1 & $3,089.3$ & 55.5 & -- & 107.7 & \\
\hline 34.44 & $3,090.6$ & 56.81 & -- & 110.4 & \\
\hline 35.6 & $3,091.8$ & 58.0 & 306.48 & 112.8 & Crest of emergency \\
\hline 36.1 & $3,092.3$ & 58.5 & -- & 196.1 & spillway. \\
\hline 36.6 & $3,092.8$ & 59.0 & -- & 352.9 & \\
\hline 37.1 & $3,093.3$ & 59.5 & - & 666.5 & \\
\hline 37.6 & $3,093.8$ & 60.0 & -- & $1,113.5$ & \\
\hline 38.6 & $3,094.8$ & 61.0 & -- & $2,334.3$ & \\
\hline 39.6 & $3,095.8$ & 62.0 & 456.12 & $4,024.3$ & \\
\hline 40.1 & $3,096.3$ & 62.5 & 478.56 & 5,060 & Top of dam. \\
\hline
\end{tabular}

1 Results of reference levels are shown in the "Supplemental Duta" section. The tie between gage height and dam height is based on levels to the crest of the principal spillway pipe inlet.

2 Computed prior to construction. 
A borrow pit in the channel upstream from the dam accounts for the first 24 acre-feet or more of stored water. The pit, as left after construction, is a few feet deeper than construction plans indicate (Wallace, 1981), and storage volumes indicated in table 1 may be in error at the lower gage heights. The borrow pit is dug into the upper layers of the aquifer (Ogallala Formation), exposing a sand, silt, clay mixture which is, in places, cemented into nodules with lime to form caliche. The caliche layer is well above the water table in this area and is so resistant to weathering that it outcrops on eroded valley sides downstream.

Investigation plans of the U.S. Soil Conservation Service (Wallace, 1981) indicate a drainage area of 8,000 acres $\left(12.5 \mathrm{mi}^{2}\right)$ for this site, of which 3,680 acres $\left(5.75 \mathrm{mi}^{2}\right)$ is uncontrolled. The uncontrolled drainage area was used to design the principal spillway pipe. The plans describe the remainder of the watershed as... "pothole country which will contribute runoff only during high intensity storms." The dam itself is about 36 feet high and about 950 feet long, not including 325 feet of emergency-spillway width.

\section{RESERVOIR WATER LEVELS}

A standard 50-foot range mercury manometer, housed in a fiberglass shelter at the north end of the dam, was used to sense water levels in the reservoir through an orifice placed in the dead-storage pool. A graphic recorder, geared to the manometer, provided a continuous record of stage for periods when the pool level rose above the orifice at a gage height of 1.36 feet. Staff gages placed at intervals in the bank of the reservoir were used to independently determine reservoir water levels.

During the period of record, August 1982 to September 1986, the reservoir filled to above the orifice only three times. Once for 14 days from April 2 to 15,1984 , another for 10 days from April 30 to May 9, 1984, and a third time, for 3 days during February 20-22, 1985. The reservoir never filled to the level of the principal (lowest) spillway during the period of record; therefore, there were no losses from the impoundment to downstream surface flow.

Hourly gage heights for the three periods of reservoir storage are given in the "Supplemental Data" section and are plotted in hydrograph form in figure 3.

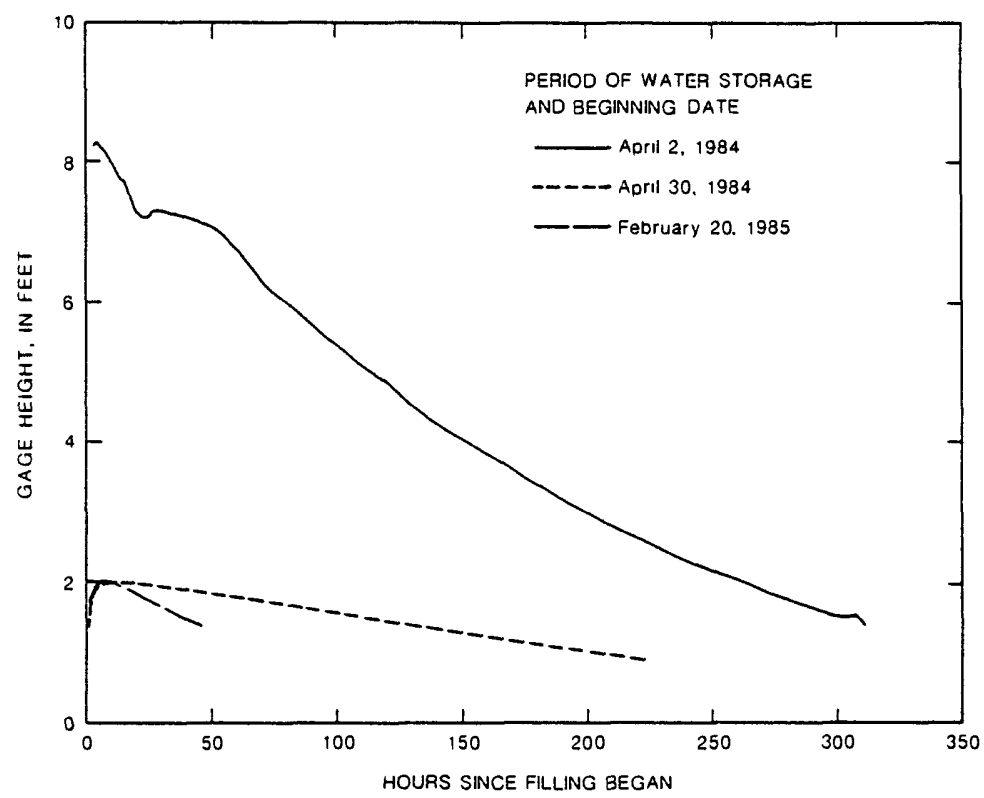

Figure 3. Reservoir gage heights during periods of water storage. 


\section{GROUND-WATER LEVELS}

Observation well 1 (fig. 2) was fitted with a recorder to continuously monitor ground-water levels at the site. The well is cased with 4 -inch polyvinyl-chloride pipe of which the lower 58.3 feet is perforated (Kelley, 1980). The driller's log and other recorded data for this well are reported in the "Supplemental Data." Initially, water levels in this well were recorded hourly by a punch-tape recorder that was driven by a float in the well. Compaction of the dam during construction caused a deflection of the upper part of the well casing, however, resulting in a poor float-to-recorder response. Because greater care in interpreting the record was necessary, the recorder was changed to a graphic continuous recorder.

Water levels in observation well 1 are shown in figure 4 for April 1 to May 31, 1984. Observation notes indicate that recorded response to water-level changes was poor during this time, and a correction was prorated from zero on day 5 (April 5) to -0.40 foot on day 39 (May 9) when the recorder was reset. The effect of this correction is an apparent gradual increase in water levels during that period, whereas the actual water-level changes may have occurred more sporadically.

Recorded water levels in observation well 1 are shown in figure 5 for every 5 days of the entire period of record.

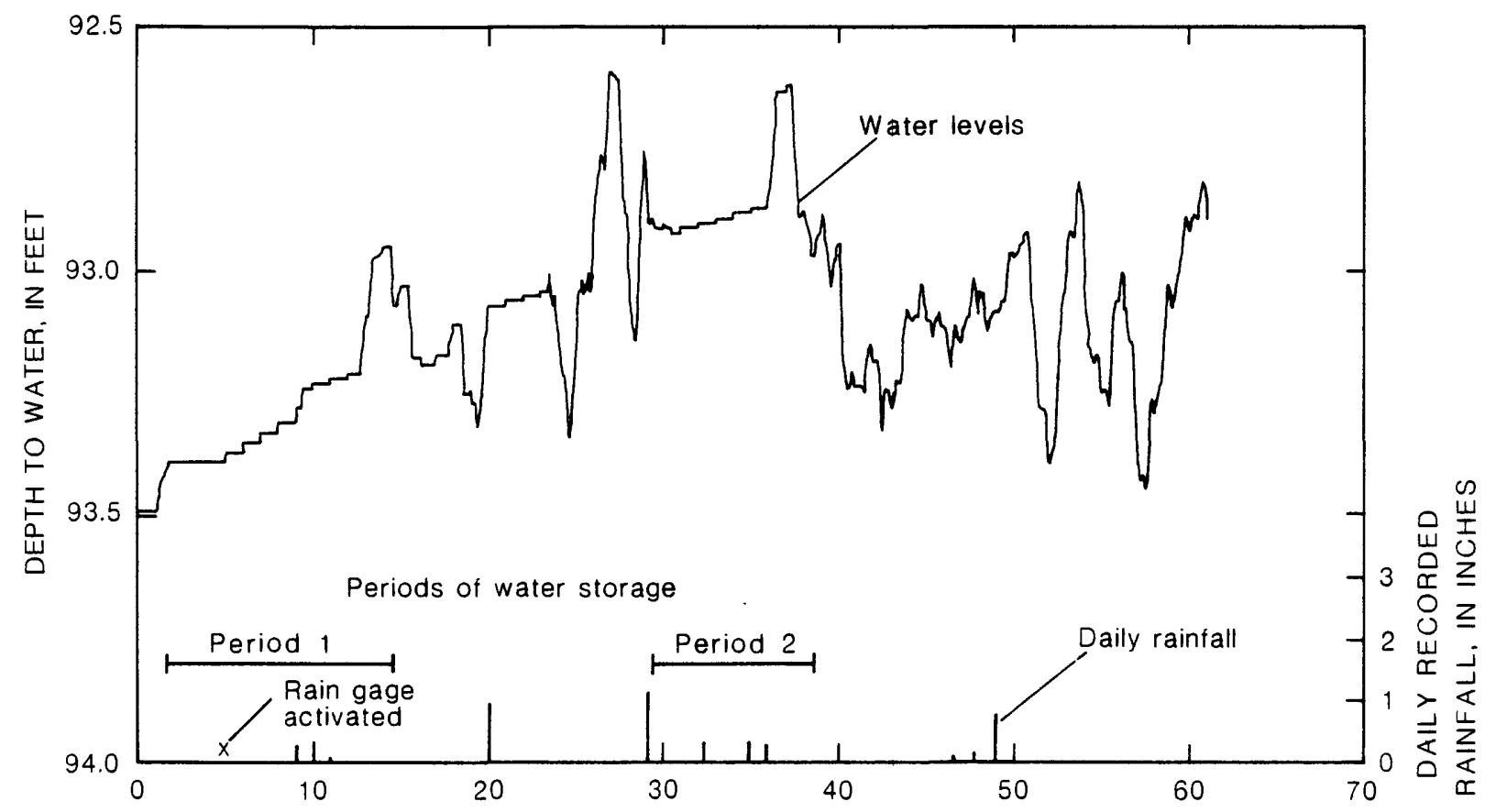

DAYS BEGINNING APRIL 1, 1984

Figure 4. Water levels in observation well 1, April 1 to May 31, 1984. 
Observation wells 2 and 3 (fig. 2) were cased with 2 -inch polyvinyl-chloride pipe. Logs for these wells are presented in the "Supplemental Data" section. Water-level measurements at these wells were made by steel tape during site visits. A borehole directly across the valley from observation well 3 was abandoned at 26 feet because of lost circulation in a lime-rich zone of the aquifer only slightly lower than the bottom of the impoundment.

Water levels in all three observation wells, as measured during site visits, are documented in the "Supplemental Data" section and graphed in figure 6.

\section{RAINFALL}

Near the reservoir manometer shelter, a separate structure supported an 8-inch rain-collector funnel (fig. 2). A punch-tape recorder, with a 15-minute interval, was housed inside the structure and was driven by a float in a container into which the rain-collector funnel drained. The U.S. Geological Survey uses a computer program to read and translate the punch tape to accumulated rainfall and rainfall-intensity values. There were no overhead obstacles near the gage to disturb the collection of rainfall. A summary showing daily rainfall data is given in the "Supplemental Data" section. A bar graph showing daily rainfall greater than 0.1 inch is shown in figure 5 for comparison with groundwater levels. The rainfall recorder was not in operation during winter months.

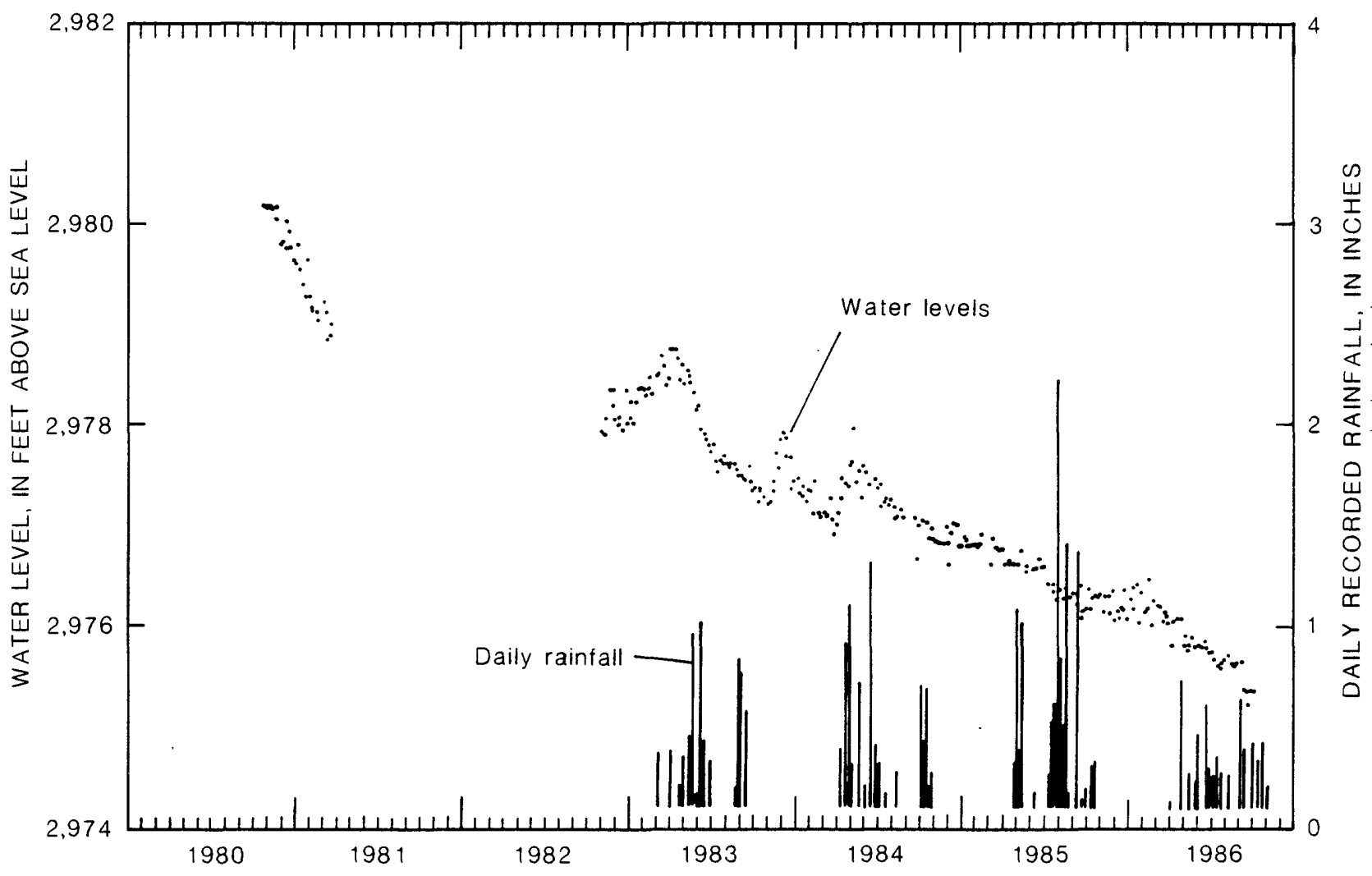

Figure 5. Five-day water levels in observation well 1 and recorded daily rainfall for period of record. 


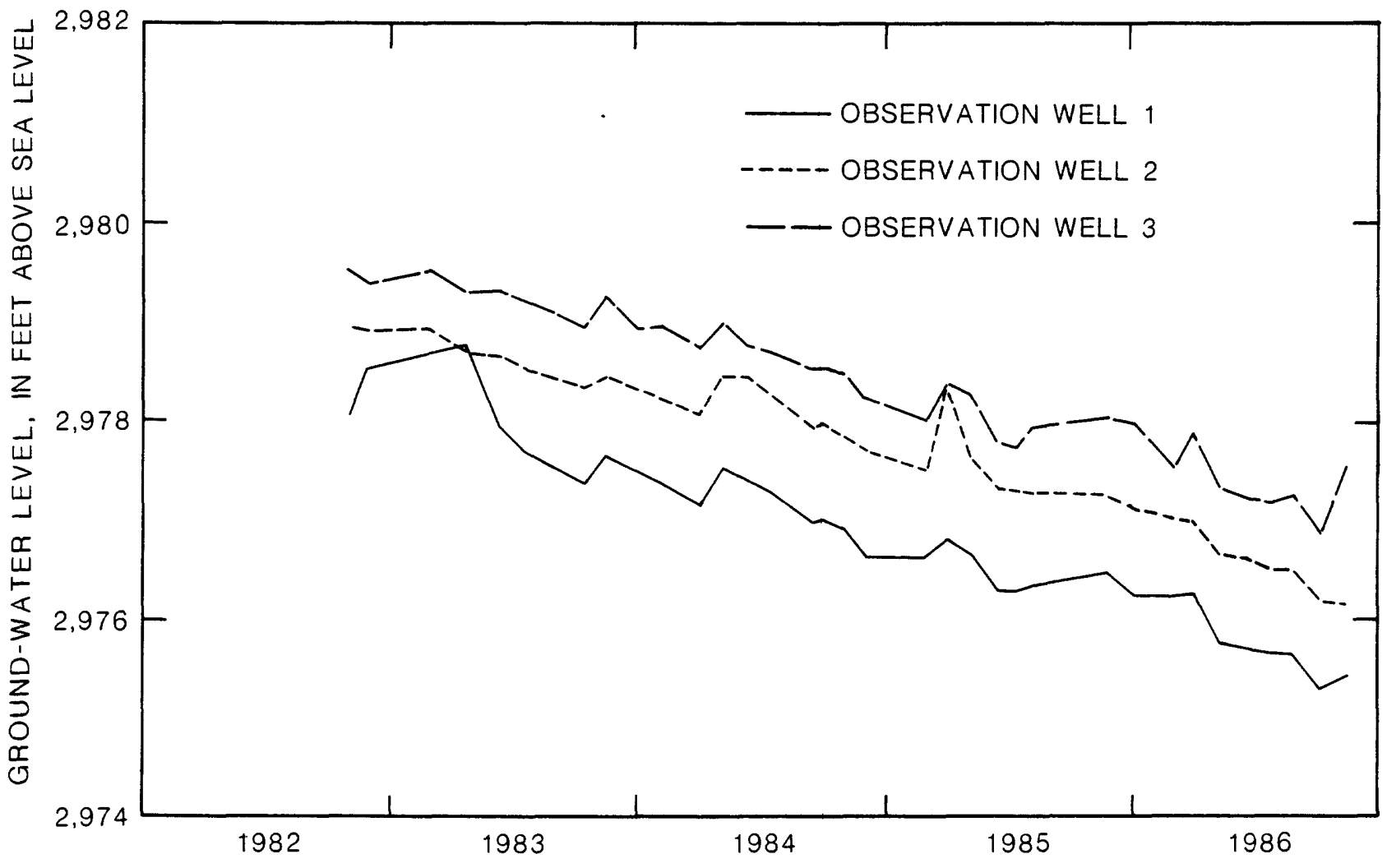

Figure 6. Water levels in observation wells at Janzen damsite.

\section{SUMMARY}

Construction of an earthen dam on a tributary to Ladder Creek in northeastern Scott County, Kansas, was sponsored by Western Kansas Groundwater Management District No. 1. The dam was built by the U.S. Soil Conservation Service (SCS) during the winter and spring of 1981-82. The dam collects surface drainage from 8,000 acres and is capable of impounding 24 acre-feet of dead storage and another 276 acre-feet of storage with uncontrolled spill through a 36 -inch metal pipe. The SCS also drilled three deep observation wells around the periphery of the impoundment into the underlying aquifer.

From August 1982 to September 1986, the U.S. Geological Survey collected rainfall, reservoirwater level, and ground-water level data at the site to document the availability of water and the recharge resulting from the water impoundment. Rainfall was recorded at 15 -minute intervals April to October each year. Reservoir pool level was monitored continuously all year as were ground-water levels in an observation well at the "toe" of the dam. Two other observation wells were measured monthly. 
The dam impounded water three times--April 2-15, 1984, April 30 to May 9, 1984, and February 20-22, 1985. All storage was contained in the dead storage pool and therefore moved from the pool by either evaporation or infiltration. The partial reservoir filling of April 2-15, 1984, was the largest documented as the reservoir filled to a gage height of 8.29 feet (about 2 acre-feet) in a 5-hour period. The reservoir then began losing water at approximately 0.7 foot per day. During the reservoir filling of April 30 to May 9, 1984, the reservoir filled to a gage height of 2.01 feet (less than 1 acre-foot) and lost water at a much slower rate of about 0.1 foot per day. On February 20, 1985, the reservoir again filled to a gage height of 2.03 feet but lost water at a rate of 0.4 foot per day.

\section{REFERENCES}

Kelley, G.A., 1980, Detailed report of geologic investigations, Keith Janzen groundwater dam: U.S. Department of Agriculture, Soil Conservation Service Communication 40-16, $46 \mathrm{p}$.

Wallace, J.A., 1981, Janzen recharge dam, Scott County, Kansas: U.S. Department of Agriculture, Soil Conservation Service Project Plans, 12 sheets. 


\section{SUPPLEMENTAL DATA}

Table 2. Hourly reservoir gage heights, April 2-15, 1984, April 30 to May 9, 1984, and February 20.22, 1984

[E indicates no record; gage height, in feet above the gage datum, is estimated based on adjacent recorded gage heights]

\begin{tabular}{|c|c|c|c|c|c|c|c|c|c|}
\hline \multirow{2}{*}{\multicolumn{2}{|c|}{$\begin{array}{l}\text { April 2,1984 } \\
\text { Time Gage } \\
\text { (24- height } \\
\text { hour) (feet) }\end{array}$}} & \multirow{2}{*}{\multicolumn{2}{|c|}{$\begin{array}{l}\text { April 3, 1984 } \\
\text { Time Gage } \\
\text { (24- height } \\
\text { hour) (feet) }\end{array}$}} & \multirow{2}{*}{\multicolumn{2}{|c|}{$\begin{array}{l}\text { April 4, 1984 } \\
\text { Time Gage } \\
\text { (24- height } \\
\text { hour) (feet) }\end{array}$}} & \multirow{2}{*}{\multicolumn{2}{|c|}{$\begin{array}{l}\text { April } 5,1984 \\
\text { Time Gage } \\
\text { (24- height } \\
\text { hour) (feet) }\end{array}$}} & \multirow{2}{*}{\multicolumn{2}{|c|}{$\begin{array}{l}\text { April 6,1984 } \\
\text { Time Gage } \\
\text { (24- height } \\
\text { hour) (feet) }\end{array}$}} \\
\hline & & & & & & & & & \\
\hline 0100 & -- & 0100 & $7.94 \mathrm{E}$ & 0100 & $7.24 \mathrm{E}$ & 0100 & $6.79 \mathrm{E}$ & 0100 & $5.88 \mathrm{E}$ \\
\hline 0200 & -- & 0200 & $7.89 \mathrm{E}$ & 0200 & $7.23 \mathrm{E}$ & 0200 & $6.76 \mathrm{E}$ & 0200 & $5.85 \mathrm{E}$ \\
\hline 0300 & -- & 0300 & $7.82 \mathrm{E}$ & 0300 & $7.22 \mathrm{E}$ & 0300 & $6.71 \mathrm{E}$ & 0300 & $5.83 \mathrm{E}$ \\
\hline 0400 & -- & 0400 & $7.74 \mathrm{E}$ & 0400 & $7.21 \mathrm{E}$ & 0400 & $6.67 \mathrm{E}$ & 0400 & $5.79 \mathrm{E}$ \\
\hline 0500 & -- & 0500 & $7.76 \mathrm{E}$ & 0500 & $7.20 \mathrm{E}$ & 0500 & $6.62 \mathrm{E}$ & 0500 & $5.76 \mathrm{E}$ \\
\hline 0600 & -- & 0600 & $7.60 \mathrm{E}$ & 0600 & $7.19 \mathrm{E}$ & 0600 & $6.58 \mathrm{E}$ & 0600 & $5.73 \mathrm{E}$ \\
\hline 0700 & -- & 0700 & $7.52 \mathrm{E}$ & 0700 & $7.18 \mathrm{E}$ & 0700 & $6.53 \mathrm{E}$ & 0700 & $5.70 \mathrm{E}$ \\
\hline 0800 & -- & 0800 & $7.44 \mathrm{E}$ & 0800 & $7.17 \mathrm{E}$ & 0800 & $6.49 \mathrm{E}$ & 0800 & $5.67 \mathrm{E}$ \\
\hline 0900 & -- & 0900 & $7.36 \mathrm{E}$ & 0900 & $7.16 \mathrm{E}$ & 0900 & $6.44 \mathrm{E}$ & 0900 & $5.64 \mathrm{E}$ \\
\hline 1000 & -- & 1000 & $7.31 \mathrm{E}$ & 1000 & $7.15 \mathrm{E}$ & 1000 & $6.39 \mathrm{E}$ & 1000 & $5.61 \mathrm{E}$ \\
\hline 1100 & -- & 1100 & 7.24 & 1100 & $7.14 \mathrm{E}$ & 1100 & 6.34 & 1100 & $5.85 \mathrm{E}$ \\
\hline 1200 & -- & 1200 & 7.19 & 1200 & 7.13 & 1200 & 6.29 & 1200 & 5.54 \\
\hline 1300 & -- & 1300 & 7.19 & 1300 & 7.12 & 1300 & 6.25 & 1300 & 5.51 \\
\hline 1400 & 1.36 & 1400 & 7.21 & 1400 & 7.11 & 1400 & 6.22 & 1400 & 5.47 \\
\hline 1500 & 1.82 & 1500 & 7.24 & 1500 & 7.10 & 1500 & 6.18 & 1500 & 5.45 \\
\hline 1600 & 1.82 & 1600 & 7.34 & 1600 & 7.08 & 1600 & 6.16 & 1600 & 5.42 \\
\hline 1700 & 8.24 & 1700 & 7.33 & 1700 & 7.06 & 1700 & 6.13 & 1700 & 5.40 \\
\hline 1800 & 8.29 & 1800 & $7.31 \mathrm{E}$ & 1800 & 7.04 & 1800 & 6.10 & 1800 & 5.37 \\
\hline 1900 & $8.26 \mathrm{E}$ & 1900 & $7.30 \mathrm{E}$ & 1900 & 7.01 & 1900 & 6.07 & 1900 & $5.34 \mathrm{E}$ \\
\hline 2000 & $8.22 \mathrm{E}$ & 2000 & $7.29 \mathrm{E}$ & 2000 & $6.97 \mathrm{E}$ & 2000 & $6.04 \mathrm{E}$ & 2000 & $5.31 \mathrm{E}$ \\
\hline 2100 & $8.19 \mathrm{E}$ & 2100 & $7.28 \mathrm{E}$ & 2100 & $6.94 \mathrm{E}$ & 2100 & $6.01 \mathrm{E}$ & 2100 & $5.28 \mathrm{E}$ \\
\hline 2200 & $8.14 \mathrm{E}$ & 2200 & $7.27 \mathrm{E}$ & 2200 & $6.91 \mathrm{E}$ & 2200 & $5.98 \mathrm{E}$ & 2200 & $5.25 \mathrm{E}$ \\
\hline 2300 & $8.09 \mathrm{E}$ & 2300 & $7.26 \mathrm{E}$ & 2300 & $6.87 \mathrm{E}$ & 2300 & $5.95 \mathrm{E}$ & 2300 & $5.23 \mathrm{E}$ \\
\hline 2400 & $8.02 \mathrm{E}$ & 2400 & $7.25 \mathrm{E}$ & 2400 & $6.82 \mathrm{E}$ & 2400 & $5.92 \mathrm{E}$ & 2400 & $5.20 \mathrm{E}$ \\
\hline
\end{tabular}


Table 2. Hourly reservoir gage heights, April 2-15, 1984, April 30 to May 9, 1984, and February 20-22, 1984--Continued

\begin{tabular}{|c|c|c|c|c|c|c|c|c|c|}
\hline \multicolumn{2}{|c|}{$\begin{array}{l}\text { April 7,1984 } \\
\text { Time Gage } \\
\text { (24- height } \\
\text { hour) (feet) }\end{array}$} & \multicolumn{2}{|c|}{$\begin{array}{l}\text { April } 8,1984 \\
\text { Time Gage } \\
(24-\text { height } \\
\text { hour) (feet) }\end{array}$} & \multicolumn{2}{|c|}{$\begin{array}{l}\text { April 9, } 1984 \\
\text { Time Gage } \\
\text { (24- height } \\
\text { hour) (feet) }\end{array}$} & \multicolumn{2}{|c|}{$\begin{array}{l}\text { April 10,1984 } \\
\text { Time Gage } \\
\text { (24- height } \\
\text { hour) (feet) }\end{array}$} & \multicolumn{2}{|c|}{$\begin{array}{l}\text { April 11,1984 } \\
\text { Time Gage } \\
\text { (24- height } \\
\text { hour) (feet) }\end{array}$} \\
\hline 0100 & $5.18 \mathrm{E}$ & 0100 & $4.47 \mathrm{E}$ & 0100 & $3.92 \mathrm{E}$ & 0100 & $3.40 \mathrm{E}$ & 0100 & $2.90 \mathrm{E}$ \\
\hline 0200 & $5.15 \mathrm{E}$ & 0200 & $4.44 \mathrm{E}$ & 0200 & $3.90 \mathrm{E}$ & 0200 & $3.38 \mathrm{E}$ & 0200 & $2.88 \mathrm{E}$ \\
\hline 0300 & $5.12 \mathrm{E}$ & 0300 & $4.42 \mathrm{E}$ & 0300 & $3.87 \mathrm{E}$ & 0300 & $3.36 \mathrm{E}$ & 0300 & $2.86 \mathrm{E}$ \\
\hline 0400 & $5.10 \mathrm{E}$ & 0400 & $4.39 \mathrm{E}$ & 0400 & $3.85 \mathrm{E}$ & 0400 & $3.34 \mathrm{E}$ & 0400 & $2.85 \mathrm{E}$ \\
\hline 0500 & $5.07 \mathrm{E}$ & 0500 & $4.37 \mathrm{E}$ & 0500 & $3.83 \mathrm{E}$ & 0500 & $3.32 \mathrm{E}$ & 0500 & $2.83 \mathrm{E}$ \\
\hline 0600 & $5.04 \mathrm{E}$ & 0600 & $4.35 \mathrm{E}$ & 0600 & $3.81 \mathrm{E}$ & 0600 & $3.30 \mathrm{E}$ & 0600 & $2.81 \mathrm{E}$ \\
\hline 0700 & $5.01 \mathrm{E}$ & 0700 & $4.32 \mathrm{E}$ & 0700 & $3.79 \mathrm{E}$ & 0700 & $3.28 \mathrm{E}$ & 0700 & $2.79 \mathrm{E}$ \\
\hline 0800 & $4.98 \mathrm{E}$ & 0800 & $4.30 \mathrm{E}$ & 0800 & $3.77 \mathrm{E}$ & 0800 & $3.25 \mathrm{E}$ & 0800 & $2.77 \mathrm{E}$ \\
\hline 0900 & $4.96 \mathrm{E}$ & 0900 & $4.28 \mathrm{E}$ & 0900 & $3.75 \mathrm{E}$ & 0900 & $3.23 \mathrm{E}$ & 0900 & $2.75 \mathrm{E}$ \\
\hline 1000 & $4.93 \mathrm{E}$ & 1000 & 4.25 & 1000 & $3.72 \mathrm{E}$ & 1000 & $3.21 \mathrm{E}$ & 1000 & $2.73 \mathrm{E}$ \\
\hline 1100 & $4.90 \mathrm{E}$ & 1100 & 4.23 & 1100 & $3.71 \mathrm{E}$ & 1100 & $3.18 \mathrm{E}$ & 1100 & 2.72 \\
\hline 1200 & $4.88 \mathrm{E}$ & 1200 & 4.21 & 1200 & $3.68 \mathrm{E}$ & 1200 & $3.16 \mathrm{E}$ & 1200 & 2.71 \\
\hline 1300 & 4.85 & 1300 & 4.18 & 1300 & $3.66 \mathrm{E}$ & 1300 & $3.14 \mathrm{E}$ & 1300 & 2.69 \\
\hline 1400 & 4.81 & 1400 & 4.15 & 1400 & $3.64 \mathrm{E}$ & 1400 & $3.12 \mathrm{E}$ & 1400 & 2.68 \\
\hline 1500 & 4.79 & 1500 & 4.13 & 1500 & $3.62 \mathrm{E}$ & 1500 & 3.10 & 1500 & 2.66 \\
\hline 1600 & 4.76 & 1600 & 4.11 & 1600 & $3.60 \mathrm{E}$ & 1600 & 3.09 & 1600 & 2.64 \\
\hline 1700 & 4.73 & 1700 & 4.08 & 1700 & $3.58 \mathrm{E}$ & 1700 & 3.06 & 1700 & 2.63 \\
\hline 1800 & $4.69 \mathrm{E}$ & 1800 & 4.06 & 1800 & $3.56 \mathrm{E}$ & 1800 & 3.04 & 1800 & 2.62 \\
\hline 1900 & $4.66 \mathrm{E}$ & 1900 & 4.04 & 1900 & $3.53 \mathrm{E}$ & 1900 & $3.02 \mathrm{E}$ & 1900 & $2.60 \mathrm{E}$ \\
\hline 2000 & $4.63 \mathrm{E}$ & 2000 & $4.02 \mathrm{E}$ & 2000 & $3.51 \mathrm{E}$ & 2000 & $3.00 \mathrm{E}$ & 2000 & $2.58 \mathrm{E}$ \\
\hline 2100 & $4.60 \mathrm{E}$ & 2100 & $4.00 \mathrm{E}$ & 2100 & $3.49 \mathrm{E}$ & 2100 & $2.98 \mathrm{E}$ & 2100 & $2.56 \mathrm{E}$ \\
\hline 2200 & $4.57 \mathrm{E}$ & 2200 & $3.98 \mathrm{E}$ & 2200 & $3.47 \mathrm{E}$ & 2200 & $2.96 \mathrm{E}$ & 2200 & $2.55 \mathrm{E}$ \\
\hline 2300 & $4.54 \mathrm{E}$ & 2300 & $3.96 \mathrm{E}$ & 2300 & $3.44 \mathrm{E}$ & 2300 & $2.94 \mathrm{E}$ & 2300 & $2.53 \mathrm{E}$ \\
\hline 2400 & $4.50 \mathrm{E}$ & 2400 & $3.94 \mathrm{E}$ & 2400 & $3.42 \mathrm{E}$ & 2400 & $2.92 \mathrm{E}$ & 2400 & $2.51 \mathrm{E}$ \\
\hline
\end{tabular}


Table 2. Hourly reservoir gage heights, April 2-15, 1984, April 30 to May 9, 1984, and February 20-22, 1984--Continued

\begin{tabular}{|c|c|c|c|c|c|c|c|}
\hline \multicolumn{2}{|c|}{ April 12, 1984} & \multicolumn{2}{|c|}{ April 13, 1984} & \multicolumn{2}{|c|}{ April 14, 1984} & \multicolumn{2}{|c|}{ April 15, 1984} \\
\hline $\begin{array}{l}\text { Time } \\
(24- \\
\text { hour })\end{array}$ & $\begin{array}{c}\text { Gage } \\
\text { height } \\
\text { (feet) }\end{array}$ & $\begin{array}{l}\text { Time } \\
(24- \\
\text { hour })\end{array}$ & $\begin{array}{l}\text { Gage } \\
\text { height } \\
\text { (feet) }\end{array}$ & $\begin{array}{l}\text { Time } \\
\text { (24- } \\
\text { hour) }\end{array}$ & $\begin{array}{l}\text { Gage } \\
\text { height } \\
\text { (feet) }\end{array}$ & $\begin{array}{l}\text { Time } \\
(24- \\
\text { hour) }\end{array}$ & $\begin{array}{c}\text { Gage } \\
\text { height } \\
\text { (feet) }\end{array}$ \\
\hline 0100 & $2.50 \mathrm{E}$ & 0100 & $2.13 \mathrm{E}$ & 0100 & $1.79 \mathrm{E}$ & 0100 & $1.52 \mathrm{E}$ \\
\hline 0200 & $2.48 \mathrm{E}$ & 0200 & $2.11 \mathrm{E}$ & 0200 & $1.78 \mathrm{E}$ & 0200 & $1.52 \mathrm{E}$ \\
\hline 0300 & $2.46 \mathrm{E}$ & 0300 & $2.09 \mathrm{E}$ & 0300 & $1.76 \mathrm{E}$ & 0300 & $1.52 \mathrm{E}$ \\
\hline 0400 & $2.44 \mathrm{E}$ & 0400 & $2.08 \mathrm{E}$ & 0400 & $1.75 \mathrm{E}$ & 0400 & $1.52 \mathrm{E}$ \\
\hline 0500 & $2.43 \mathrm{E}$ & 0500 & $2.07 \mathrm{E}$ & 0500 & $1.74 \mathrm{E}$ & 0500 & $1.52 \mathrm{E}$ \\
\hline 0600 & $2.42 \mathrm{E}$ & 0600 & $2.05 \mathrm{E}$ & 0600 & $1.73 \mathrm{E}$ & 0600 & $1.52 \mathrm{E}$ \\
\hline 0700 & $2.40 \mathrm{E}$ & 0700 & $2.04 \mathrm{E}$ & 0700 & $1.72 \mathrm{E}$ & 0700 & $1.52 \mathrm{E}$ \\
\hline 0800 & $2.38 \mathrm{E}$ & 0800 & $2.02 \mathrm{E}$ & 0800 & $1.71 \mathrm{E}$ & 0800 & $1.52 \mathrm{E}$ \\
\hline 0900 & $2.36 \mathrm{E}$ & 0900 & $2.01 \mathrm{E}$ & 0900 & $1.70 \mathrm{E}$ & 0900 & $1.52 \mathrm{E}$ \\
\hline 1000 & 2.35 & 1000 & $2.00 \mathrm{E}$ & 1000 & $1.68 \mathrm{E}$ & 1000 & 1.41 \\
\hline 1100 & 2.33 & 1100 & 1.98 & 1100 & 1.67 & 1100 & 1.40 \\
\hline 1200 & 2.31 & 1200 & 1.97 & 1200 & 1.66 & 1200 & $1.39 \mathrm{E}$ \\
\hline 1300 & 2.30 & 1300 & 1.96 & 1300 & 1.65 & 1300 & - \\
\hline 1400 & 2.29 & 1400 & 1.95 & 1400 & 1.64 & 1400 & -- \\
\hline 1500 & 2.28 & 1500 & 1.94 & 1500 & 1.63 & 1500 & -- \\
\hline 1600 & 2.26 & 1600 & 1.93 & 1600 & 1.62 & 1600 & -- \\
\hline 1700 & 2.25 & 1700 & 1.91 & 1700 & 1.60 & 1700 & -- \\
\hline 1800 & 2.23 & 1800 & 1.89 & 1800 & 1.59 & 1800 & -- \\
\hline 1900 & $2.21 \mathrm{E}$ & 1900 & $1.88 \mathrm{E}$ & 1900 & $1.58 \mathrm{E}$ & 1900 & -- \\
\hline 2000 & $2.20 \mathrm{E}$ & 2000 & $1.87 \mathrm{E}$ & 2000 & $1.57 \mathrm{E}$ & 2000 & -- \\
\hline 2100 & $2.18 \mathrm{E}$ & 2100 & $1.85 \mathrm{E}$ & 2100 & $1.55 \mathrm{E}$ & 2100 & -- \\
\hline 2200 & $2.16 \mathrm{E}$ & 2200 & $1.84 \mathrm{E}$ & 2200 & $1.54 \mathrm{E}$ & 2000 & -- \\
\hline 2300 & $2.15 \mathrm{E}$ & 2300 & $1.82 \mathrm{E}$ & 2300 & $1.53 \mathrm{E}$ & 2300 & -- \\
\hline 2400 & $2.14 \mathrm{E}$ & 2400 & $1.81 \mathrm{E}$ & 2400 & $1.52 \mathrm{E}$ & 2400 & -. \\
\hline
\end{tabular}


Table 2. Hourly reservoir gage heights, April 2-15, April 30 to May 9, 1984, and February 20-22, 1984--Continued

\begin{tabular}{|c|c|c|c|c|c|c|c|c|c|}
\hline \multicolumn{2}{|c|}{$\begin{array}{l}\text { April } 30,1984 \\
\text { Time Gage } \\
\text { (24- height } \\
\text { hour) (feet) }\end{array}$} & $\begin{array}{l}\text { May } 1 \\
\text { Time } \\
(24- \\
\text { hour })\end{array}$ & $\begin{array}{l}\frac{1,1984}{\text { Gage }} \\
\text { height } \\
\text { (feet) }\end{array}$ & $\begin{array}{l}\text { May } 2 \\
\text { Time } \\
(24- \\
\text { hour })\end{array}$ & $\begin{array}{l}\frac{2,1984}{\text { Gage }} \\
\text { height } \\
\text { (feet) }\end{array}$ & $\begin{array}{l}\text { May } \\
\text { Time } \\
(24- \\
\text { hour })\end{array}$ & $\begin{array}{l}\frac{3,1984}{\text { Gage }} \\
\text { height } \\
\text { (feet) }\end{array}$ & $\begin{array}{l}\text { May } \\
\text { Time } \\
(24- \\
\text { hour })\end{array}$ & $\begin{array}{l}\frac{4,1984}{\text { Gage }} \\
\text { height } \\
\text { (feet) }\end{array}$ \\
\hline 0100 & -- & 0100 & $2.00 \mathrm{E}$ & 0100 & $1.88 \mathrm{E}$ & 0100 & $1.76 \mathrm{E}$ & 0100 & $1.63 \mathrm{E}$ \\
\hline 0200 & -- & 0200 & $1.99 \mathrm{E}$ & 0200 & $1.87 \mathrm{E}$ & 0200 & $1.75 \mathrm{E}$ & 0200 & $1.62 \mathrm{E}$ \\
\hline 0300 & -- & 0300 & $1.99 \mathrm{E}$ & 0300 & $1.87 \mathrm{E}$ & 0300 & $1.75 \mathrm{E}$ & 0300 & $1.61 \mathrm{E}$ \\
\hline 0400 & -- & 0400 & $1.98 \mathrm{E}$ & 0400 & $1.86 \mathrm{E}$ & 0400 & $1.74 \mathrm{E}$ & 0400 & $1.61 \mathrm{E}$ \\
\hline 0500 & -. & 0500 & $1.98 \mathrm{E}$ & 0500 & $1.86 \mathrm{E}$ & 0500 & $1.74 \mathrm{E}$ & 0500 & $1.60 \mathrm{E}$ \\
\hline 0600 & -- & 0600 & $1.97 \mathrm{E}$ & 0600 & $1.85 \mathrm{E}$ & 0600 & $1.74 \mathrm{E}$ & 0600 & $1.60 \mathrm{E}$ \\
\hline 0700 & -- & 0700 & $1.97 \mathrm{E}$ & 0700 & $1.85 \mathrm{E}$ & 0700 & $1.73 \mathrm{E}$ & 0700 & $1.59 \mathrm{E}$ \\
\hline 0800 & -- & 0800 & $1.96 \mathrm{E}$ & 0800 & $1.84 \mathrm{E}$ & 0800 & $1.73 \mathrm{E}$ & 0800 & $1.59 \mathrm{E}$ \\
\hline 0900 & $1.37 \mathrm{E}$ & 0900 & $1.95 \mathrm{E}$ & 0900 & $1.84 \mathrm{E}$ & 0900 & $1.72 \mathrm{E}$ & 0900 & $1.58 \mathrm{E}$ \\
\hline 1000 & 1.87 & 1000 & $1.95 \mathrm{E}$ & 1000 & 1.84 & 1000 & $1.72 \mathrm{E}$ & 1000 & $1.57 \mathrm{E}$ \\
\hline 1100 & 1.90 & 1100 & $1.94 \mathrm{E}$ & 1100 & 1.83 & 1100 & 1.72 & 1100 & 1.57 \\
\hline 1200 & 1.93 & 1200 & 1.94 & 1200 & 1.82 & 1200 & 1.71 & 1200 & 1.56 \\
\hline 1300 & 1.95 & 1300 & 1.93 & 1300 & 1.82 & 1300 & 1.71 & 1300 & 1.55 \\
\hline 1400 & 1.98 & 1400 & 1.93 & 1400 & $1.81 \mathrm{E}$ & 1400 & 1.70 & 1400 & 1.55 \\
\hline 1500 & 1.99 & 1500 & 1.93 & 1500 & $1.81 \mathrm{E}$ & 1500 & 1.69 & 1500 & 1.54 \\
\hline 1600 & 2.00 & 1600 & 1.92 & 1600 & $1.80 \mathrm{E}$ & 1600 & 1.69 & 1600 & 1.54 \\
\hline 1700 & 2.01 & 1700 & 1.92 & 1700 & $1.80 \mathrm{E}$ & 1700 & 1.69 & 1700 & 1.53 \\
\hline 1800 & 2.01 & 1800 & $1.91 \mathrm{E}$ & 1800 & $1.79 \mathrm{E}$ & 1800 & 1.68 & 1800 & 1.52 \\
\hline 1900 & 2.01 & 1900 & $1.91 \mathrm{E}$ & 1900 & $1.79 \mathrm{E}$ & 1900 & 1.67 & 1900 & 1.51 \\
\hline 2000 & 2.01 & 2000 & $1.91 \mathrm{E}$ & 2000 & $1.78 \mathrm{E}$ & 2000 & $1.66 \mathrm{E}$ & 2000 & $1.50 \mathrm{E}$ \\
\hline 2100 & 2.01 & 2100 & $1.90 \mathrm{E}$ & 2100 & $1.78 \mathrm{E}$ & 2100 & $1.65 \mathrm{E}$ & 2100 & $1.50 \mathrm{E}$ \\
\hline 2200 & 2.01 & 2200 & $1.89 \mathrm{E}$ & 2200 & $1.78 \mathrm{E}$ & 2200 & $1.65 \mathrm{E}$ & 2200 & $1.49 \mathrm{E}$ \\
\hline 2300 & $2.00 \mathrm{E}$ & 2300 & $1.89 \mathrm{E}$ & 2300 & $1.77 \mathrm{E}$ & 2300 & $1.64 \mathrm{E}$ & 2300 & $1.49 \mathrm{E}$ \\
\hline 2400 & $2.00 \mathrm{E}$ & 2400 & $1.88 \mathrm{E}$ & 2400 & $1.77 \mathrm{E}$ & 2400 & $1.64 \mathrm{E}$ & 2400 & $1.48 \mathrm{E}$ \\
\hline
\end{tabular}


Table 2. Hourly reservoir gage heights, April 2-15, April 30 to May 9, 1984, and February 20-22, 1984--Continued

\begin{tabular}{|c|c|c|c|c|c|c|c|c|c|}
\hline \multicolumn{2}{|c|}{$\begin{array}{l}\text { May } 5,1984 \\
\text { Time Gage } \\
\text { (24- height } \\
\text { hour) (feet) }\end{array}$} & $\begin{array}{l}\text { May } \\
\text { Time } \\
(24- \\
\text { hour })\end{array}$ & $\begin{array}{l}\frac{6,1984}{\text { Gage }} \\
\text { height } \\
\text { (feet) }\end{array}$ & $\begin{array}{l}\text { May } \\
\text { Time } \\
(24- \\
\text { hour })\end{array}$ & $\begin{array}{l}\frac{7.1984}{\text { Gage }} \\
\text { height } \\
\text { (feet) }\end{array}$ & $\begin{array}{l}\text { May } \\
\text { Time } \\
(24- \\
\text { hour })\end{array}$ & $\begin{array}{l}\frac{8,1984}{\text { Gage }} \\
\text { height } \\
\text { (feet) }\end{array}$ & $\begin{array}{l}\text { May } \\
\text { Time } \\
(24- \\
\text { hour) }\end{array}$ & $\begin{array}{l}9,1984 \\
\text { Gage } \\
\text { height } \\
\text { (feet) }\end{array}$ \\
\hline 0100 & $1.47 \mathrm{E}$ & 0100 & $1.34 \mathrm{E}$ & 0100 & 1.22 & 0100 & $1.10 \mathrm{E}$ & 0100 & $0.97 \mathrm{E}$ \\
\hline 0200 & $1.47 \mathrm{E}$ & 0200 & $1.33 \mathrm{E}$ & 0200 & 1.21 & 0200 & $1.09 \mathrm{E}$ & 0200 & $.97 \mathrm{E}$ \\
\hline 0300 & $1.46 \mathrm{E}$ & 0300 & $1.33 \mathrm{E}$ & 0300 & 1.21 & 0300 & $1.08 \mathrm{E}$ & 0300 & $.96 \mathrm{E}$ \\
\hline 0400 & $1.46 \mathrm{E}$ & 0400 & $1.32 \mathrm{E}$ & 0400 & 1.20 & 0400 & $1.08 \mathrm{E}$ & 0400 & $.96 \mathrm{E}$ \\
\hline 0500 & $1.45 \mathrm{E}$ & 0500 & $1.32 \mathrm{E}$ & 0500 & 1.20 & 0500 & $1.07 \mathrm{E}$ & 0500 & $.95 \mathrm{E}$ \\
\hline 0600 & $1.45 \mathrm{E}$ & 0600 & $1.31 \mathrm{E}$ & 0600 & 1.19 & 0600 & $1.07 \mathrm{E}$ & 0600 & $.95 \mathrm{E}$ \\
\hline 0700 & $1.44 \mathrm{E}$ & 0700 & $1.31 \mathrm{E}$ & 0700 & 1.19 & 0700 & $1.06 \mathrm{E}$ & 0700 & $.94 \mathrm{E}$ \\
\hline 0800 & $1.44 \mathrm{E}$ & 0800 & $1.30 \mathrm{E}$ & 0800 & 1.18 & 0800 & $1.06 \mathrm{E}$ & 0800 & $.94 \mathrm{E}$ \\
\hline 0900 & $1.43 \mathrm{E}$ & 0900 & $1.30 \mathrm{E}$ & 0900 & 1.17 & 0900 & $1.05 \mathrm{E}$ & 0900 & $.93 \mathrm{E}$ \\
\hline 1000 & $1.43 \mathrm{E}$ & 1000 & $1.29 \mathrm{E}$ & 1000 & 1.17 & 1000 & $1.05 \mathrm{E}$ & 1000 & $.93 \mathrm{E}$ \\
\hline 1100 & $1.42 \mathrm{E}$ & 1100 & $1.29 \mathrm{E}$ & 1100 & 1.16 & 1100 & $1.04 \mathrm{E}$ & 1100 & $.92 \mathrm{E}$ \\
\hline 1200 & 1.41 & 1200 & $1.28 \mathrm{E}$ & 1200 & 1.16 & 1200 & $1.04 \mathrm{E}$ & 1200 & $.92 \mathrm{E}$ \\
\hline 1300 & $1.40 \mathrm{E}$ & 1300 & $1.28 \mathrm{E}$ & 1300 & 1.16 & 1300 & $1.03 \mathrm{E}$ & 1300 & $.91 \mathrm{E}$ \\
\hline 1400 & $1.39 \mathrm{E}$ & 1400 & $1.27 \mathrm{E}$ & 1400 & 1.15 & 1400 & $1.03 \mathrm{E}$ & 1400 & $.91 \mathrm{E}$ \\
\hline 1500 & $1.39 \mathrm{E}$ & 1500 & $1.27 \mathrm{E}$ & 1500 & 1.15 & 1500 & $1.02 \mathrm{E}$ & 1500 & .90 \\
\hline 1600 & $1.38 \mathrm{E}$ & 1600 & $1.26 \mathrm{E}$ & 1600 & 1.14 & 1600 & $1.02 \mathrm{E}$ & 1600 & -- \\
\hline 1700 & $1.37 \mathrm{E}$ & 1700 & $1.26 \mathrm{E}$ & 1700 & 1.13 & 1700 & $1.01 \mathrm{E}$ & 1700 & -- \\
\hline 1800 & $1.37 \mathrm{E}$ & 1800 & $1.25 \mathrm{E}$ & 1800 & 1.13 & 1800 & $1.01 \mathrm{E}$ & 1800 & -- \\
\hline 1900 & $1.37 \mathrm{E}$ & 1900 & $1.25 \mathrm{E}$ & 1900 & 1.12 & 1900 & $1.00 \mathrm{E}$ & 1900 & -- \\
\hline 2000 & $1.36 \mathrm{E}$ & 2000 & $1.24 \mathrm{E}$ & 2000 & 1.12 & 2000 & $1.00 \mathrm{E}$ & 2000 & -- \\
\hline 2100 & $1.36 \mathrm{E}$ & 2100 & $1.24 \mathrm{E}$ & 2100 & 1.11 & 2100 & $.99 \mathrm{E}$ & 2100 & -- \\
\hline 2200 & $1.35 \mathrm{E}$ & 2200 & $1.23 \mathrm{E}$ & 2200 & 1.11 & 2200 & $.99 \mathrm{E}$ & 2200 & -- \\
\hline 2300 & $1.35 \mathrm{E}$ & 2300 & $1.23 \mathrm{E}$ & 2300 & 1.10 & 2300 & $.98 \mathrm{E}$ & 2300 & -- \\
\hline 2400 & $1.34 \mathrm{E}$ & 2400 & $1.22 \mathrm{E}$ & 2400 & 1.10 & 2400 & $.98 \mathrm{E}$ & 2400 & -- \\
\hline
\end{tabular}


Table 2. Hourly reservoir gage heights, April 2-15, April 30 to May 9, 1984, and February 20-22, 1984--Continued

\begin{tabular}{|c|c|c|c|c|c|}
\hline \multicolumn{2}{|c|}{$\begin{array}{c}\text { February } 20 \\
1985\end{array}$} & \multicolumn{2}{|c|}{$\begin{array}{c}\text { February } 21 \\
1985\end{array}$} & \multicolumn{2}{|c|}{$\begin{array}{c}\text { February } 22, \\
1985\end{array}$} \\
\hline $\begin{array}{l}\text { Time } \\
(24- \\
\text { hour })\end{array}$ & $\begin{array}{l}\text { Gage } \\
\text { height } \\
\text { (feet) }\end{array}$ & $\begin{array}{l}\text { Time } \\
(24- \\
\text { hour) }\end{array}$ & $\begin{array}{c}\text { Gage } \\
\text { height } \\
\text { (feet) }\end{array}$ & $\begin{array}{l}\text { Time } \\
(24- \\
\text { hour) }\end{array}$ & $\begin{array}{c}\text { Gage } \\
\text { height } \\
\text { (feet) }\end{array}$ \\
\hline 0100 & -- & 0100 & 1.98 & 0100 & 1.55 \\
\hline 0200 & -- & 0200 & 1.96 & 0200 & 1.53 \\
\hline 0300 & -- & 0300 & 1.94 & 0300 & 1.51 \\
\hline 0400 & -- & 0400 & 1.92 & 0400 & 1.49 \\
\hline 0500 & -- & 0500 & 1.90 & 0500 & 1.47 \\
\hline 0600 & -- & 0600 & 1.89 & 0600 & 1.46 \\
\hline 0700 & -- & 0700 & 1.87 & 0700 & 1.44 \\
\hline 0800 & -- & 0800 & 1.86 & 0800 & 1.42 \\
\hline 0900 & -. & 0900 & 1.84 & 0900 & 1.41 \\
\hline 1000 & -- & 1000 & 1.82 & 1000 & 1.39 \\
\hline 1100 & -- & 1100 & 1.80 & 1100 & 1.37 \\
\hline 1200 & -- & 1200 & 1.78 & 1200 & -- \\
\hline 1300 & -- & 1300 & 1.76 & 1300 & -- \\
\hline 1400 & 1.37 & 1400 & 1.75 & 1400 & -- \\
\hline 1500 & 1.65 & 1500 & 1.73 & 1500 & -- \\
\hline 1600 & 1.85 & 1600 & 1.71 & 1600 & -- \\
\hline 1700 & 1.95 & 1700 & 1.69 & 1700 & .- \\
\hline 1800 & 2.01 & 1800 & 1.67 & 1800 & -- \\
\hline 1900 & 2.03 & 1900 & 1.66 & 1900 & -- \\
\hline 2000 & 2.03 & 2000 & 1.64 & 2000 & -- \\
\hline 2100 & 2.03 & 2100 & 1.63 & 2100 & -. \\
\hline 2200 & 2.01 & 2200 & 1.61 & 2200 & -- \\
\hline 2300 & 1.99 & 2300 & 1.59 & 2300 & -- \\
\hline 2400 & 1.98 & 2400 & 1.57 & 2400 & -- \\
\hline
\end{tabular}


Table 3. Hourly recorded depth to water in observation well 1, April 1 to May 31,1984 [Depths to water are in feet below land surface]

Date Time

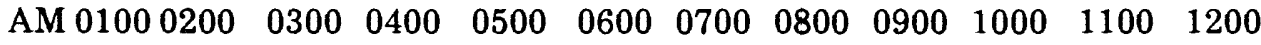

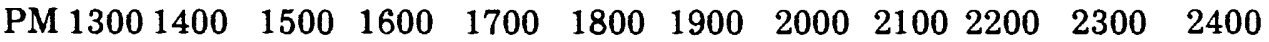

Apr. $\quad 93.4893 .4893 .4893 .4893 .4893 .4893 .4893 .4893 .4893 .4893 .4993 .49$

193.4993 .4993 .4993 .4993 .4993 .4993 .4993 .4993 .4993 .4993 .4993 .49

Apr. $\quad 93.4993 .4993 .4893 .4793 .4493 .4493 .4393 .4293 .4293 .4293 .4293 .41$

$2 \quad 93.4193 .4093 .4093 .3993 .3993 .3993 .3993 .3993 .3993 .3993 .3993 .39$

Apr. $\quad 93.3993 .3993 .3993 .3993 .3993 .3993 .3993 .3993 .3993 .3993 .3993 .39$

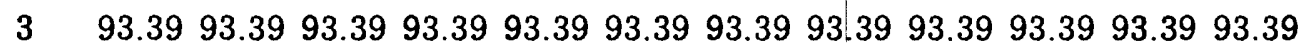

Apr. $\quad 93.3993 .3993 .3993 .3993 .3993 .3993 .3993 .34 \quad 33.3993 .3993 .3993 .39$

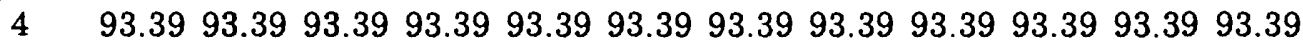

Apr. $\quad 93.3993 .3993 .3993 .3993 .3993 .3993 .3993 .3993 .3993 .3993 .3993 .39$

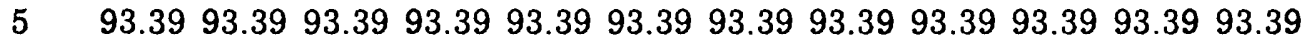

Apr. $\quad 93.3793 .3793 .3793 .3793 .3793 .3793 .3793 .3793 .3793 .3793 .3793 .37$

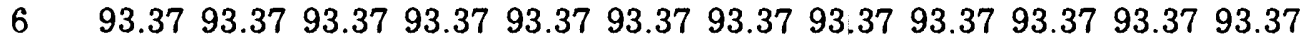

Apr. $\quad 93.3593 .3593 .3593 .3593 .3593 .3593 .3593 .3593 .3593 .3593 .3593 .35$

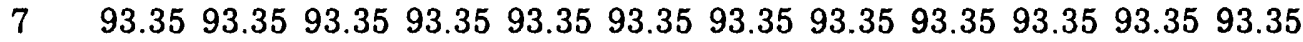

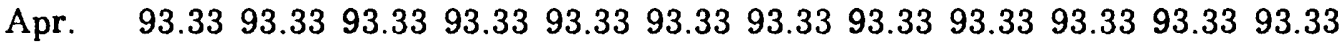

$\begin{array}{lllllllllllll}8 & 93.33 & 93.33 & 93.33 & 93.33 & 93.33 & 93.33 & 93.33 & 93.33 & 93.33 & 93.33 & 93.33 & 93.33\end{array}$

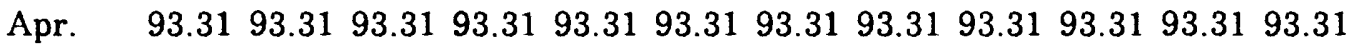

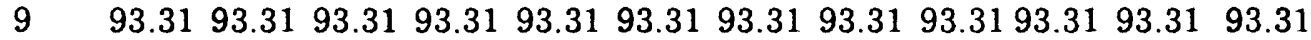

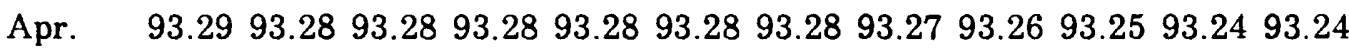

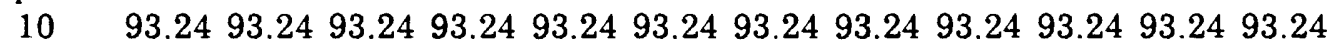

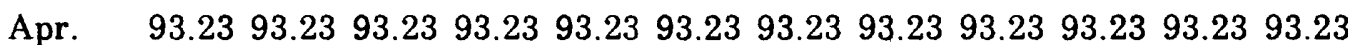

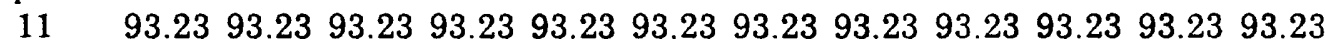

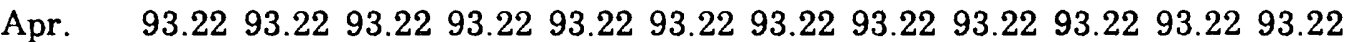

$12 \quad 93.22 \quad 93.22 \quad 93.2293 .2293 .2293 .2293 .2293 .2293 .2293 .2293 .2293 .22$

Apr. $\quad 93.2193 .2193 .2193 .2193 .2193 .2193 .2193 .2193 .2193 .2193 .2193 .21$

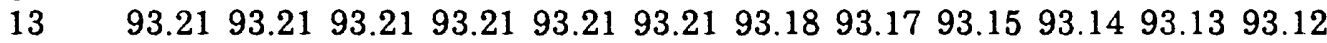

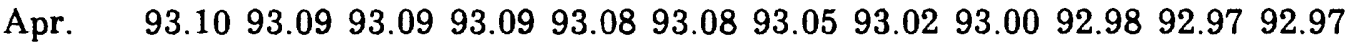
$14 \quad 92.9792 .9792 .9792 .9792 .9792 .9792 .9792 .9792 .9692 .9692 .9692 .96$

Apr. $\quad 92.9592 .9592 .9592 .9592 .9592 .9592 .9592 .9592 .9592 .9592 .9692 .98$ $15 \quad 93.0093 .0293 .0593 .0793 .0793 .0793 .0793 .0793 .0693 .0493 .0493 .04$ 
Table 3. Hourly recorded depth to water in observation well 1, April 1 to May 31,1984-Continued

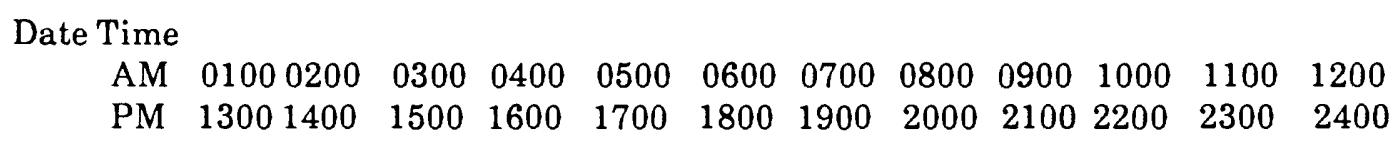

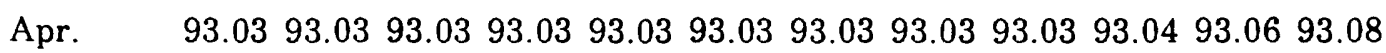

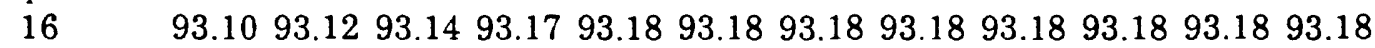

Apr. $\quad 93.1793 .1793 .1793 .1793 .1993 .1993 .1993 .1993 .1993 .1993 .1993 .19$

$17 \quad 93.1993 .1993 .1993 .1993 .1993 .1993 .1993 .1993 .1993 .1993 .1993 .18$

Apr. $\quad 93.1793 .1793 .1793 .1793 .1793 .1793 .1793 .1793 .1793 .1793 .1793 .17$

$18 \quad 93.1793 .1793 .1793 .1793 .1793 .1793 .1793 .1593 .1493 .1493 .1393 .13$

Apr. $\quad 93.1193 .1193 .1193 .1193 .1193 .1193 .1193 .1193 .1193 .1193 .1193 .12$

$19 \quad 93.1493 .1893 .2293 .23 \quad 93.2593 .2593 .2593 .2593 .2593 .2593 .2593 .25$

Apr. $\quad 93.2493 .26 \quad 93.27 \quad 93.27 \quad 93.2793 .2793 .2793 .2793 .3093 .3293 .3193 .31$

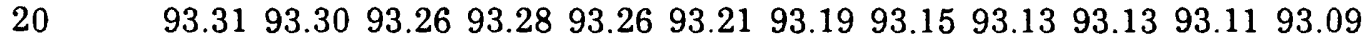

Apr. $\quad 93.0793 .0793 .0793 .0793 .0793 .0793 .0793 .0793 .0793 .0793 .0793 .07$

$21 \quad 93.0793 .0793 .0793 .0793 .0793 .0793 .0793 .0793 .0793 .0793 .0793 .07$

Apr. $\quad 93.0693 .0693 .0693 .0693 .0693 .0693 .0693 .0693 .0693 .0693 .0693 .06$

$22 \quad 93.0693 .0693 .0693 .0693 .0693 .0693 .0693 .0693 .0693 .0693 .0693 .06$

Apr. $\quad 93.0593 .0593 .0593 .0593 .0593 .0593 .0593 .0593 .0593 .0593 .0593 .05$

$23 \quad 93.05 \quad 93.0593 .0593 .0593 .05 \quad 93.0593 .05 \quad 93.0593 .0593 .0593 .0593 .05$

Apr. $\quad 93.0493 .0493 .0493 .0493 .0493 .0493 .0493 .0493 .0493 .0493 .0493 .04$

$24 \quad 93.0093 .0093 .0393 .0593 .0693 .0793 .06 \quad 93.0593 .0593 .0593 .0793 .10$

Apr. $\quad 93.1193 .1393 .1493 .1693 .1993 .2193 .2193 .2193 .2193 .2393 .2393 .25$

$25 \quad 93.26 \quad 93.2893 .3193 .3493 .3493 .33 \quad 93.3293 .2993 .2493 .2293 .1993 .12$

Apr. $\quad 93.0993 .0693 .0493 .0493 .0493 .0493 .0293 .0193 .0193 .0293 .0493 .05$

$26 \quad 93.0593 .0493 .0493 .0193 .0093 .0293 .0493 .0493 .0493 .0293 .0292 .97$

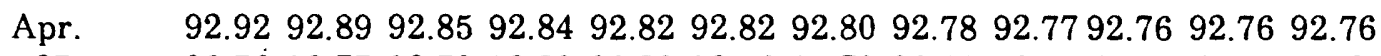

$27 \quad 92.7692 .7792 .7892 .7992 .7892 .7692 .7392 .6892 .6492 .6192 .6192 .60$

Apr. $\quad 92.5992 .5992 .6092 .6092 .6092 .6192 .6192 .6192 .6192 .6192 .6492 .68$

$\begin{array}{lllllllllllll}28 & 92.74 & 92.76 & 92.79 & 92.83 & 92.85 & 92.86 & 92.88 & 92.88 & 92.88 & 92.88 & 92.90 & 92.91\end{array}$

Apr. $\quad 92.9693 .0093 .0393 .0493 .0593 .0993 .1193 .0993 .1493 .1393 .1393 .13$

$29 \quad 93.0693 .0292 .9792 .9392 .8992 .8592 .8492 .8192 .7792 .7592 .7792 .79$

Apr. $\quad 92.8092 .8492 .8892 .9092 .9092 .9092 .9092 .9092 .9092 .8992 .8992 .90$

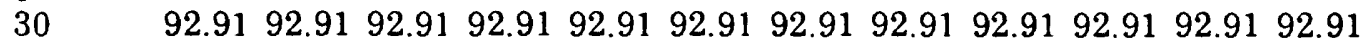


Table 3. Hourly recorded depth to water in observation well 1, April 1 to May 31, 1984-Continued

Date Time

$\begin{array}{lllllllllll}\text { AM } 01000200 & 0300 & 0400 & 0500 & 0600 & 0700 & 0800 & 0900 & 1000 & 1100 & 1200\end{array}$

PM $13001400 \quad 1500 \quad 1600 \quad 1700 \quad 1800 \quad 1900 \quad 2000 \quad 2100 \quad 2200 \quad 2300 \quad 2400$

May $\quad 92.90 \quad 92.90 \quad 92.90 \quad 92.9092 .91 \quad 92.9192 .9192 .9192 .9192 .9192 .9192 .91$

$1 \quad 92.92 \quad 92.92 \quad 92.92 \quad 92.92 \quad 92.9292 .9292 .92 \quad 92.92 \quad 92.92 \quad 92.92 \quad 92.9292 .92$

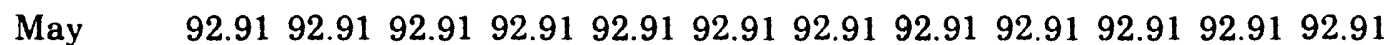

$2 \quad 92.9192 .9192 .9192 .9192 .9192 .9192 .9192 .9192 .9192 .9192 .9192 .91$

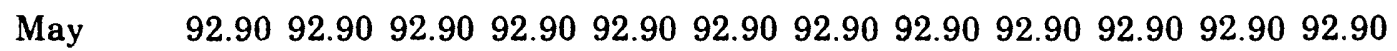

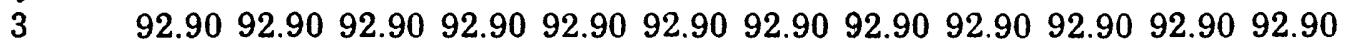

May $\quad 92.8992 .8992 .8992 .8992 .8992 .8992 .8992 .8992 .8992 .8992 .8992 .89$

$4 \quad 92.8992 .8992 .8992 .8992 .8992 .8992 .8992 .8992 .8992 .8992 .8992 .89$

$\begin{array}{llllllllllllllll}\text { May } & \quad & 92.88 & 92.88 & 92.88 & 92.88 & 92.88 & 92.88 & 92.88 & 92.88 & 92.88 & 92.88 & 92.88 & 92.88\end{array}$

$\begin{array}{lllllllllllll}5 & 92.88 & 92.88 & 92.88 & 92.88 & 92.88 & 92.88 & 92.88 & 92.88 & 92.88 & 92.88 & 92.88 & 92.88\end{array}$

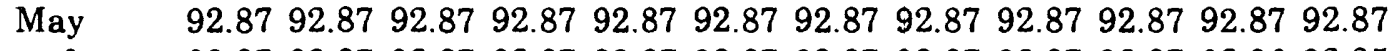

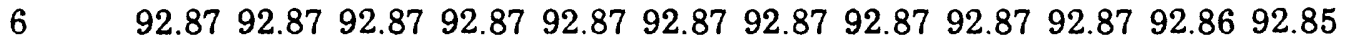

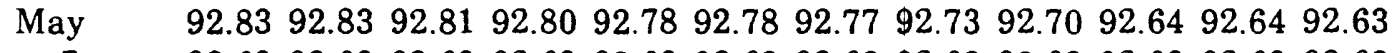

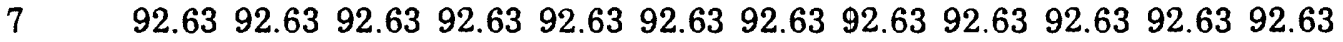

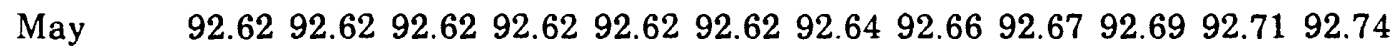

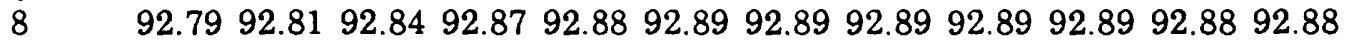

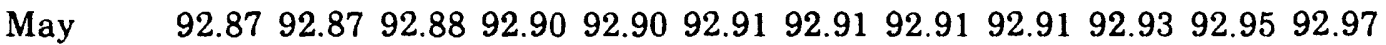

$9 \quad 92.9792 .9792 .9792 .9792 .9692 .9492 .9392 .9392 .9292 .9292 .9292 .92$

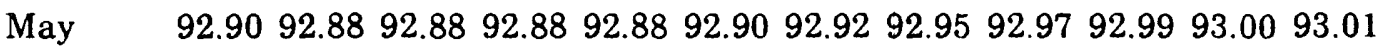

$10 \quad 93.0293 .0393 .0393 .03 \quad 93.0293 .0192 .9892 .9792 .9792 .9792 .9792 .95$

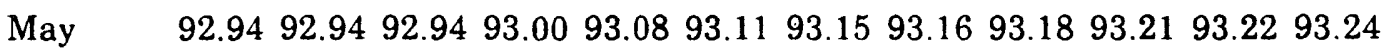

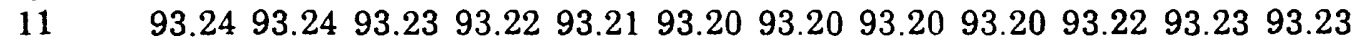

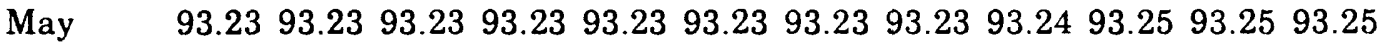

$12 \quad 93.25 \quad 93.23 \quad 93.2093 .1893 .1793 .1693 .16 \quad 93.1693 .1593 .1793 .1893 .18$

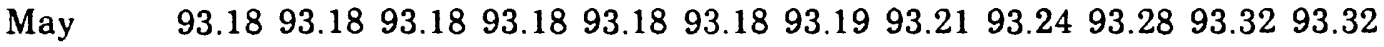

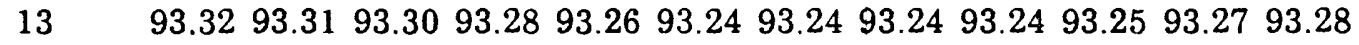


Table 3. Hourly recorded depth to water in observation well 1, April 1 to May 31,1984-Continued

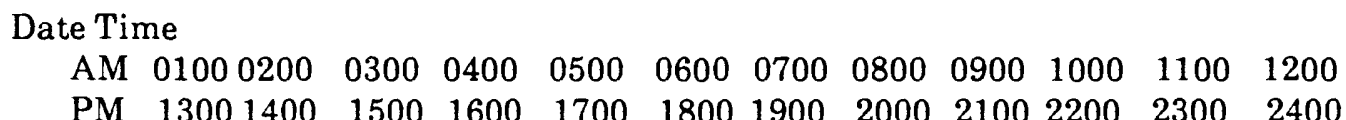

$\begin{array}{lllllllllllll}\text { May } & 92.28 & 93.27 & 93.27 & 93.26 & 93.25 & 93.23 & 93.22 & 93.22 & 93.23 & 93.23 & 93.23 & 93.23\end{array}$

$14 \quad 93.2193 .1893 .1693 .1393 .1193 .1093 .0993 .0893 .0893 .0893 .0893 .09$

May $\quad 93.0993 .0993 .0993 .1093 .1093 .0993 .0993 .0993 .0993 .0993 .0993 .09$

15993.0893 .0593 .0593 .0493 .0393 .0293 .0293 .0293 .0393 .0593 .0793 .08

May $\quad 93.0993 .1093 .1093 .1093 .1093 .1093 .1193 .1293 .1393 .1393 .1293 .12$

$16 \quad 93.1193 .0993 .1093 .0993 .0993 .0893 .0893 .0993 .0993 .1193 .1193 .11$

May $\quad 93.1293 .1293 .1293 .1293 .1393 .1493 .1593 .1793 .1993 .1993 .1993 .19$

$17 \quad 93.1793 .1693 .1593 .1293 .1293 .1193 .1293 .1393 .1393 .1493 .1493 .14$

May $\quad 93.1493 .1493 .1293 .1293 .1193 .1093 .1093 .1093 .0993 .0993 .0993 .09$

18993.0993 .0793 .0693 .0493 .0193 .0193 .0193 .0293 .0493 .0593 .0893 .09

May $\quad 93.0793 .0593 .0493 .0493 .0493 .0593 .0593 .0893 .1093 .1193 .1193 .12$

$19 \quad 93.1293 .1293 .1293 .1093 .0993 .0993 .0893 .0893 .0893 .0893 .0893 .08$

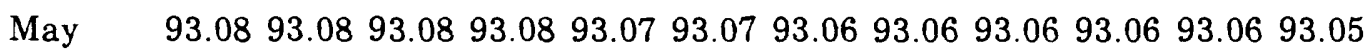

$20 \quad 93.0493 .03 \quad 93.0193 .0092 .9992 .9792 .9692 .9692 .9692 .9692 .9692 .97$

May $\quad 92.9792 .9792 .9792 .9792 .9692 .9692 .9592 .9592 .9592 .9592 .9492 .94$

$21 \quad 92.9492 .9492 .9492 .9292 .9292 .9292 .9292 .9292 .9492 .9993 .0393 .04$

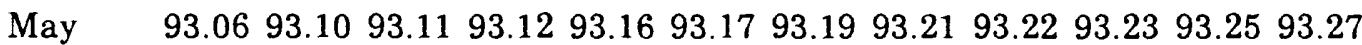

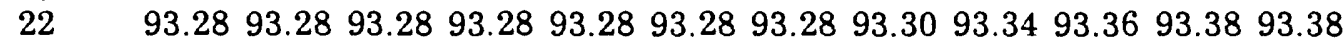

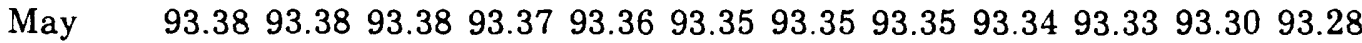

$23 \quad 93.2593 .2193 .1793 .1493 .1193 .0993 .0693 .0593 .0493 .0393 .0393 .03$

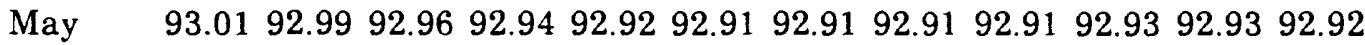
$24 \quad 92.9292 .9192 .8992 .8792 .8392 .8292 .8192 .8192 .8492 .8592 .9193 .01$

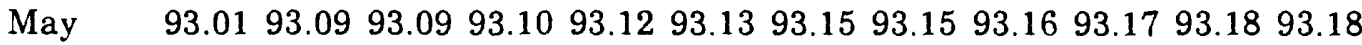

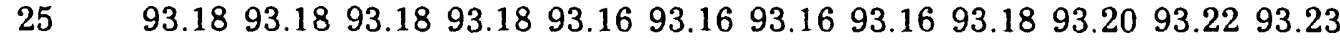

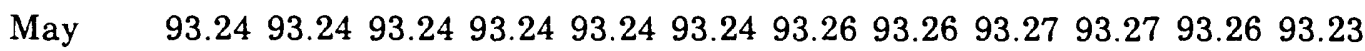
$26 \quad 93.2193 .1893 .1493 .13 \quad 93.1093 .0793 .0693 .0693 .0693 .0693 .0693 .05$

May $\quad 93.0493 .0393 .0293 .0093 .0093 .0093 .0793 .0793 .0793 .1093 .1193 .11$ $27 \quad 93.1293 .1393 .1493 .1493 .1493 .1493 .1593 .1693 .2093 .2593 .3293 .34$ 
Table 3. Hourly recorded depth to water in observation well 1, April 1 to May 31, 1984-. Continued

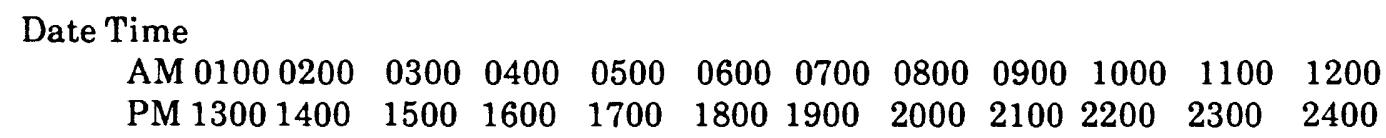

May $\quad 93.3793 .3993 .4093 .4193 .4293 .4193 .4193 .4193 .43 \quad 93.4493 .4493 .44$

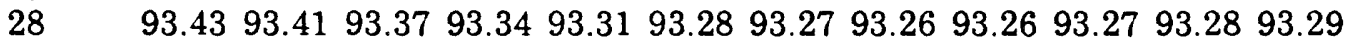

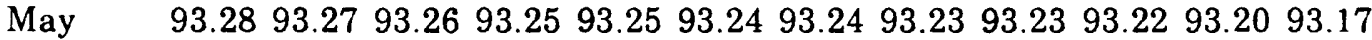

$29 \quad 93.1493 .1193 .0993 .0793 .0593 .03 \quad 93.02 \quad 93.0293 .0293 .0393 .0593 .07$

May $\quad 93.0793 .0793 .0693 .0493 .0493 .0393 .0293 .0293 .0193 .0092 .9992 .99$

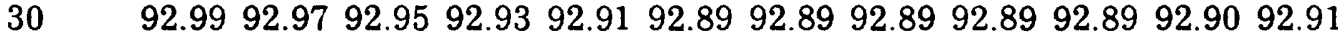

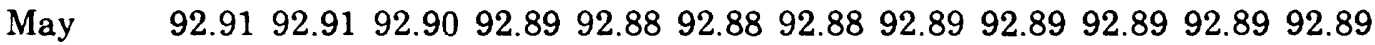

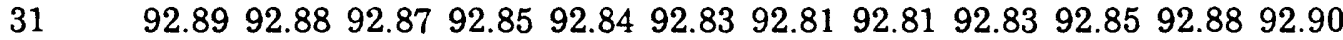


Table 4. Five-day recorded depths to water in observation well 1

\begin{tabular}{|c|c|c|c|c|c|c|c|c|c|c|}
\hline $\begin{array}{c}\text { Date } \\
\text { (month } \\
\text { day/ } \\
\text { year) }\end{array}$ & $\begin{array}{l}\text { Depth } \\
\text { to } \\
\text { water } \\
\text { (feet) }\end{array}$ & $\begin{array}{c}\text { Date } \\
\text { (month } \\
\text { day/ } \\
\text { year) }\end{array}$ & $\begin{array}{l}\text { Depth } \\
\text { to } \\
\text { water } \\
\text { (feet) }\end{array}$ & $\begin{array}{c}\text { Date } \\
\text { (month } \\
\text { day/ } \\
\text { year) }\end{array}$ & $\begin{array}{l}\text { Depth } \\
\text { to } \\
\text { water } \\
\text { (feet) }\end{array}$ & $\begin{array}{c}\text { Date } \\
\text { (month } \\
\text { day/ } \\
\text { year) }\end{array}$ & $\begin{array}{l}\text { Depth } \\
\text { to } \\
\text { water } \\
\text { (feet) }\end{array}$ & $\begin{array}{c}\text { Date } \\
\text { (month } \\
\text { day/ } \\
\text { year) }\end{array}$ & $\begin{array}{l}\text { Depth } \\
\text { to } \\
\text { water } \\
\text { (feet) }\end{array}$ & $\begin{array}{l}\text { Date Depth } \\
\text { (month to } \\
\text { day/ water } \\
\text { year) (feet) }\end{array}$ \\
\hline
\end{tabular}

$10 / 25 / 8087.57$ $10 / 30 / 8087.57$ $11 / 05 / 8087.59$ $11 / 10 / 8087.57$ $11 / 15 / 8087.60$

$11 / 20 / 8087.70$ $11 / 25 / 8087.58$ $11 / 30 / 8087.96$ $12 / 05 / 8087.92$ $12 / 10 / 8087.72$

$12 / 15 / 8087.99$ $12 / 20 / 8087.82$ $12 / 25 / 8087.98$ $12 / 30 / 8088.11$ $01 / 05 / 8188.14$

$01 / 10 / 8187.95$ $01 / 15 / 8188.20$ $01 / 20 / 8188.36$ $01 / 25 / 8188.48$ $01 / 30 / 8188.11$

$02 / 05 / 81 \quad 88.48$ $02 / 10 / 8188.59$ $02 / 15 / 8188.63$ $02 / 20 / 8188.62$ $02 / 25 / 8188.71$

$03 / 05 / 8188.51$ $03 / 10 / 8188.64$ $03 / 15 / 8188.90$ $03 / 20 / 8188.86$ $03 / 24 / 8188.75$
$02 / 20 / 8394.28$ $02 / 25 / 8394.45$ $03 / 05 / 8394.26$ $03 / 10 / 8394.24$ $03 / 15 / 8394.07$

$03 / 20 / 8394.17$ $03 / 25 / 8394.36$ $03 / 30 / 8394.28$ $04 / 05 / 8394.00$ $04 / 10 / 8394.00$

$04 / 15 / 8394.00$ $04 / 20 / 8394.08$ $04 / 25 / 8394.31$ $04 / 30 / 8391.92^{1}$ $05 / 05 / 8392.11$

$05 / 10 / 8391.97$ $05 / 15 / 8392.03$ $05 / 20 / 8392.11$ $05 / 25 / 8392.20$ $05 / 30 / 8392.37$

$06 / 05 / 8392.33$ $06 / 10 / 8392.56$ $06 / 15 / 8392.60$ $06 / 20 / 8392.66$ $06 / 25 / 8392.72$

$06 / 30 / 8392.78$ $07 / 05 / 8392.71$ $07 / 10 / 8392.87$ $07 / 15 / 8392.98$ $07 / 20 / 8392.86$

$11 / 05 / 8294.82^{1,207 / 25 / 8392.90}$ $11 / 10 / 8294.85 \quad 07 / 30 / 8392.83$ $11 / 15 / 8294.70 \quad 08 / 05 / 8392.91$ $11 / 20 / 8294.40 \quad 08 / 10 / 8392.94$ $11 / 25 / 8294.40 \quad 08 / 15 / 8392.91$
$11 / 10 / 8393.18$ $11 / 15 / 8393.08$ $11 / 20 / 8392.79$ $11 / 25 / 8392.94$ $11 / 30 / 8392.66$

$12 / 05 / 8392.60$ $12 / 10 / 8392.83$ $12 / 15 / 8392.65$ $12 / 20 / 8392.85$ $12 / 25 / 8393.14$

$12 / 30 / 8393.06$ $01 / 05 / 8493.05$ $01 / 10 / 8493.19$ $01 / 15 / 8493.22$ $01 / 20 / 8493.14$

$01 / 25 / 8493.27$ $01 / 30 / 8493.16$ $02 / 05 / 8493.17$ $02 / 10 / 8493.39$ $02 / 15 / 8493.08$

$02 / 20 / 8493.39$ $02 / 25 / 8493.43$ $03 / 05 / 8493.40$ $03 / 10 / 8493.42$ $03 / 15 / 8493.24$

$03 / 20 / 8493.46$ $03 / 25 / 8493.60$ $03 / 30 / 8493.49$ $04 / 05 / 8493.39$ $04 / 10 / 8493.24$

$\begin{array}{llll}04 / 15 / 84 & 93.04 & 01 / 20 / 85 & 93.72\end{array}$ $04 / 20 / 8493.09$ $04 / 25 / 8493.12$ $04 / 30 / 8492.91$ $05 / 05 / 8492.88$
$07 / 30 / 8493.25$ $08 / 05 / 8493.33$ $08 / 10 / 8493.43$ $08 / 15 / 8493.42$ $08 / 20 / 8493.36$

$08 / 25 / 8493.43$ $09 / 20 / 8493.44$ $09 / 25 / 8493.85$ $09 / 30 / 8493.50$ $10 / 05 / 8493.47$

$10 / 10 / 8493.48$ $10 / 15 / 8493.49$ $10 / 20 / 8493.65$ $10 / 25 / 84 \quad 93.55$ $10 / 30 / 8493.66$

$11 / 05 / 8493.69$ $11 / 10 / 8493.70$ $11 / 15 / 8493.70$ $11 / 20 / 8493.70$ $11 / 25 / 8493.53$

$11 / 30 / 8493.70$ $12 / 05 / 8493.90$ $12 / 10 / 8493.59$ $12 / 15 / 8493.50$ $12 / 20 / 8493.51$

$12 / 25 / 8493.72$ $12 / 30 / 8493.72$ $01 / 05 / 8593.63$ $01 / 10 / 8593.67$ $01 / 15 / 8593.71$

$01 / 25 / 8593.70$ $01 / 30 / 8593.71$ $02 / 05 / 8593.73$ $02 / 10 / 8593.71$
05/20/85 93.97 05/25/85 93.92 $05 / 30 / 8593.95$ $06 / 05 / 8593.94$ $06 / 10 / 8593.94$

$06 / 15 / 8593.94$ $06 / 20 / 8593.85$ $06 / 25 / 8593.93$ $06 / 30 / 8593.93$ $07 / 05 / 8594.10$

$07 / 10 / 8594.11$ $07 / 15 / 8594.17$ $07 / 20 / 8594.11$ $07 / 25 / 8594.26$ $07 / 30 / 8594.15$

$08 / 05 / 8594.16$ $08 / 10 / 8594.24$ $08 / 15 / 8594.14$ $08 / 20 / 8594.20$ $08 / 25 / 8594.23$

$08 / 30 / 8594.19$ $09 / 05 / 8594.20$ $09 / 10 / 8594.32$ $09 / 15 / 8594.12$ 09/20/85 94.44

$09 / 25 / 8594.36$ $09 / 30 / 8594.35$ $10 / 05 / 8594.16$ $10 / 10 / 8594.35$ $10 / 15 / 8594.23$

$10 / 20 / 8594.21$ $10 / 25 / 8594.23$ $10 / 30 / 8594.20$ $11 / 05 / 8594.38$ $11 / 10 / 8594.22$
$02 / 05 / 86 \quad 94.37$ $02 / 05 / 86 \quad 94.37$ $02 / 10 / 8694.34$ $02 / 15 / 8694.07$ $02 / 20 / 8694.51$

$02 / 25 / 8694.21$ $03 / 05 / 8694.32$ $03 / 10 / 8694.33$ $03 / 15 / 8694.47$ $03 / 20 / 8694.38$

$03 / 25 / 86 \quad 94.42$ $03 / 30 / 8694.49$ $04 / 05 / 8694.71$ $04 / 10 / 8694.47$ $04 / 15 / 8694.45$

$04 / 20 / 86 \quad 94: 44$ $04 / 25 / 8694.44$ $04 / 30 / 8694.71$ $05 / 05 / 8694.63$ $05 / 10 / 8694.76$

$05 / 15 / 8694.70$ $05 / 20 / 8694.64$ $05 / 25 / 8694.73$ $05 / 30 / 8694.71$ $06 / 05 / 8694.72$

$06 / 10 / 8694.73$ $06 / 15 / 8694.68$ $06 / 20 / 8694.75$ $06 / 25 / 8694.79$ $06 / 30 / 8694.77$

$07 / 05 / 8694.78$ $07 / 10 / 8694.85$ $07 / 15 / 8694.90$ $07 / 20 / 8694.94$ $07 / 25 / 8694.88$ 
Table 4. Five-day recorded depths to water in observation well 1--Continued

\begin{tabular}{|c|c|c|c|c|c|c|c|c|c|c|}
\hline $\begin{array}{c}\text { Date } \\
\text { (month } \\
\text { day/ } \\
\text { year) }\end{array}$ & $\begin{array}{l}\text { Depth } \\
\text { to } \\
\text { water } \\
\text { (feet) }\end{array}$ & $\begin{array}{c}\text { Date } \\
\text { (month } \\
\text { day/ } \\
\text { year) }\end{array}$ & $\begin{array}{l}\text { Depth } \\
\text { to } \\
\text { water } \\
\text { (feet) }\end{array}$ & $\begin{array}{c}\text { Date } \\
\text { (month } \\
\text { day/ } \\
\text { year) }\end{array}$ & $\begin{array}{l}\text { Depth } \\
\text { to } \\
\text { water } \\
\text { (feet) }\end{array}$ & $\begin{array}{c}\text { Date } \\
\text { (month } \\
\text { day/ } \\
\text { year) }\end{array}$ & $\begin{array}{l}\text { Depth } \\
\text { to } \\
\text { water } \\
\text { (feet) }\end{array}$ & $\begin{array}{c}\text { Date } \\
\text { (month } \\
\text { day/ } \\
\text { year) }\end{array}$ & $\begin{array}{l}\text { Depth } \\
\text { to } \\
\text { water } \\
\text { (feet) }\end{array}$ & $\begin{array}{l}\text { Date Depth } \\
\text { (month to } \\
\text { day/ water } \\
\text { year) (feet) }\end{array}$ \\
\hline $11 / 30 / 82$ & 94.56 & $08 / 20 / 83$ & 92.91 & $05 / 10 / 84$ & 92.55 & $02 / 15 / 85$ & 93.61 & $11 / 15 / 85$ & 94.22 & $07 / 30 / 8694.85$ \\
\hline $12 / 05 / 82$ & 94.70 & $08 / 25 / 83$ & 92.96 & $05 / 15 / 84$ & 93.08 & $03 / 05 / 85$ & 93.90 & $11 / 20 / 85$ & 594.40 & 08/05/86 94.82 \\
\hline $12 / 10 / 82$ & 94.76 & $08 / 30 / 83$ & 93.02 & $05 / 20 / 84$ & 92.97 & $03 / 10 / 85$ & 93.64 & $11 / 25 / 85$ & 594.18 & $08 / 10 / 8694.90$ \\
\hline $12 / 15 / 82$ & 94.68 & $09 / 05 / 83$ & 93.02 & $05 / 25 / 84$ & 93.23 & $03 / 15 / 85$ & 93.74 & $11 / 30 / 85$ & 594.45 & $08 / 15 / 8694.88$ \\
\hline $12 / 20 / 82$ & 294.81 & $09 / 10 / 83$ & 93.05 & $05 / 30 / 84$ & 92.91 & $03 / 20 / 85$ & 93.75 & $12 / 05 / 85$ & 94.37 & $08 / 20 / 8694.91$ \\
\hline $12 / 25 / 82$ & 294.41 & $09 / 15 / 83$ & 93.06 & $06 / 05 / 84$ & 92.99 & $03 / 25 / 85$ & 93.76 & $12 / 10 / 85$ & 594.45 & $08 / 25 / 8694.88$ \\
\hline $12 / 30 / 82$ & 94.73 & $09 / 20 / 83$ & 92.93 & $06 / 10 / 84$ & 93.11 & $03 / 30 / 85$ & 93.75 & $12 / 15 / 85$ & 594.35 & $08 / 30 / 8694.88$ \\
\hline $01 / 05 / 83$ & 94.68 & $09 / 25 / 83$ & 93.09 & $06 / 15 / 84$ & 93.10 & $04 / 05 / 85$ & 93.90 & $12 / 20 / 85$ & 94.36 & 09/05/86 94.88 \\
\hline $01 / 10 / 83$ & 94.53 & $09 / 30 / 83$ & 93.16 & $06 / 20 / 84$ & 93.06 & $04 / 10 / 85$ & 93.87 & $12 / 25 / 85$ & 594.17 & $09 / 10 / 8695.15$ \\
\hline $01 / 15 / 83$ & 94.73 & $10 / 05 / 83$ & $93.14^{1}$ & $06 / 25 / 84$ & 93.05 & $04 / 15 / 85$ & 93.87 & $12 / 30 / 85$ & 594.45 & $09 / 15 / 8695.17$ \\
\hline $01 / 20 / 83$ & 3 94.52 & $10 / 10 / 83$ & 93.27 & $06 / 30 / 84$ & 93.15 & $04 / 20 / 85$ & 93.90 & $01 / 05 / 86$ & 694.25 & $09 / 20 / 8695.30$ \\
\hline $01 / 25 / 83$ & 394.39 & $10 / 15 / 83$ & 93.16 & $07 / 05 / 84$ & 93.11 & $04 / 25 / 85$ & 93.90 & $01 / 10 / 86$ & 694.35 & $09 / 25 / 8695.17$ \\
\hline $01 / 30 / 83$ & 94.38 & $10 / 20 / 83$ & 93.22 & $07 / 10 / 84$ & 93.32 & $04 / 30 / 85$ & 93.90 & $01 / 15 / 86$ & 694.13 & $09 / 30 / 8695.17$ \\
\hline $02 / 05 / 83$ & 94.40 & $10 / 25 / 83$ & 93.22 & $07 / 15 / 84$ & 93.27 & $05 / 05 / 85$ & 93.91 & $01 / 20 / 86$ & 694.11 & \\
\hline $02 / 10 / 83$ & 394.45 & $10 / 30 / 83$ & 93.30 & $07 / 20 / 84$ & 93.22 & $05 / 10 / 85$ & 93.77 & $01 / 25 / 86$ & 694.49 & \\
\hline $02 / 15 / 83$ & 394.39 & $11 / 05 / 83$ & 93.28 & $07 / 25 / 84$ & 93.31 & $05 / 15 / 85$ & 93.97 & $01 / 30 / 86$ & 694.21 & \\
\hline
\end{tabular}

1 Measuring point changed at this time.

2 No record March 24, 1981, to November 2, 1982. 
Table 5. Measurement of depths to water in observation wells 1,2, and 3 made during site visits

\begin{tabular}{|c|c|c|}
\hline $\begin{array}{l}\text { Date } \\
\text { (month/ } \\
\text { day/ } \\
\text { year) }\end{array}$ & $\begin{array}{c}\text { Depth } \\
\text { to water } \\
\text { (feet) }\end{array}$ & Remarks \\
\hline
\end{tabular}

05/30/80 87.99--Measuring point $=$ top of casing at $3,067.74$ feet 07/24/80 87.33 above sea level, 4.2 feet above ground level.

10/22/80 87.63--Installed analog-to-digital (punch-tape) recorder $11 / 21 / 80 \quad 87.57$ (12-hour intervals).

$12 / 24 / 80 \quad 87.69$

03/24/81 88.78--Removed analog-to-digital (punch-tape) recorder. $04 / 29 / 81 \quad 88.25$

$06 / 19 / 81 \quad 88.26$

$08 / 20 / 81 \quad 88.96$

$09 / 30 / 81 \quad 88.72$

$11 / 05 / 81 \quad 88.85$

$01 / 20 / 82$ 95.43--Measuring point $=$ top of casing at 3,074.74 feet above sea level, 11.2 feet above ground level (added 7 feet to casing).

$03 / 20 / 82 \quad 95.77$

$05 / 10 / 82 \quad 95.90$

$08 / 02 / 82 \quad 96.12$

$11 / 02 / 8294.72-$-Measuring point $=$ top of casing at $3,072.74$ feet $12 / 03 / 8294.22$ above sea level; installed analog-to-digital (punch-tape) recorder (1-hour interval).

03/01/83 196.22--Total depth noted on analog-to-digital recorder. $03 / 01 / 83189.54-$-Measuring point $=$ hole in casing at $3,070.52$ feet $04 / 27 / 8391.74$ above sea level.

$06 / 16 / 83 \quad 92.61$

$07 / 20 / 83 \quad 92.86$

$08 / 23 / 83 \quad 92.98$

$10 / 17 / 83 \quad 93.17$

$11 / 18 / 83 \quad 92.87$

$01 / 06 / 84 \quad 93.06$

$02 / 07 / 84 \quad 93.16$

$04 / 05 / 84 \quad 93.39$

$05 / 09 / 84 \quad 92.99$

$06 / 12 / 84 \quad 93.12$ 
Table 5. Measurement of depths to water in observation wells 1, 2, and 3 made during site visits --Continued

\begin{tabular}{|c|c|c|}
\hline $\begin{array}{l}\text { Date } \\
\text { (month/ } \\
\text { day/ } \\
\text { year) }\end{array}$ & $\begin{array}{l}\text { Depth } \\
\text { to water } \\
\text { (feet) }\end{array}$ & Remarks \\
\hline
\end{tabular}

07/16/84 93.24--Analog-to-digital (punch-tape) recorder replaced 09/18/84 93.57 with strip recorder, 08/28/84.

10/02/84 93.53--Measuring point $=$ top of casing at $3,070.52$ feet

above sea level, well slug tested, okay.

$11 / 02 / 84 \quad 93.63$

$12 / 04 / 84 \quad 93.90$

$03 / 04 / 85 \quad 93.90$

$04 / 04 / 85 \quad 93.72$

$05 / 09 / 85 \quad 93.88$

$06 / 18 / 85 \quad 94.24$

$07 / 15 / 85 \quad 94.26$

$08 / 05 / 85194.19$

$10 / 09 / 85 \quad 94.80$

$11 / 25 / 85 \quad 94.05$

$01 / 06 / 86 \quad 94.29$

$03 / 06 / 86 \quad 94.31$

$04 / 02 / 86 \quad 94.27$

$05 / 12 / 86 \quad 94.78$

$06 / 23 / 86 \quad 94.84$

$07 / 25 / 86 \quad 94.87$

08/29/86 94.88

$10 / 06 / 86 \quad 95.24$

$11 / 13 / 86 \quad 95.11$ 
Table 5. Measurement of depths to water in observation wells 1, 2, and 3 made during site visits--Continued

\begin{tabular}{ccc}
$\begin{array}{c}\text { Date } \\
\text { (month/ } \\
\text { day/ } \\
\text { year) }\end{array}$ & $\begin{array}{c}\text { Depth } \\
\text { to water } \\
\text { (feet) }\end{array}$ & Well 2 \\
\hline
\end{tabular}

$06 / 19 / 81 \quad 100.52-$-Measuring point $=$ top of casing at $3,080.88$ feet above $08 / 20 / 81101.30$ sea level, 0.5 foot at ground level.

$11 / 05 / 81 \quad 100.99$

$01 / 20 / 82101.00$-Measuring point $=$ top of casing at $3,080.38$ feet above 03/20/82 99.83 sea level, at ground level.

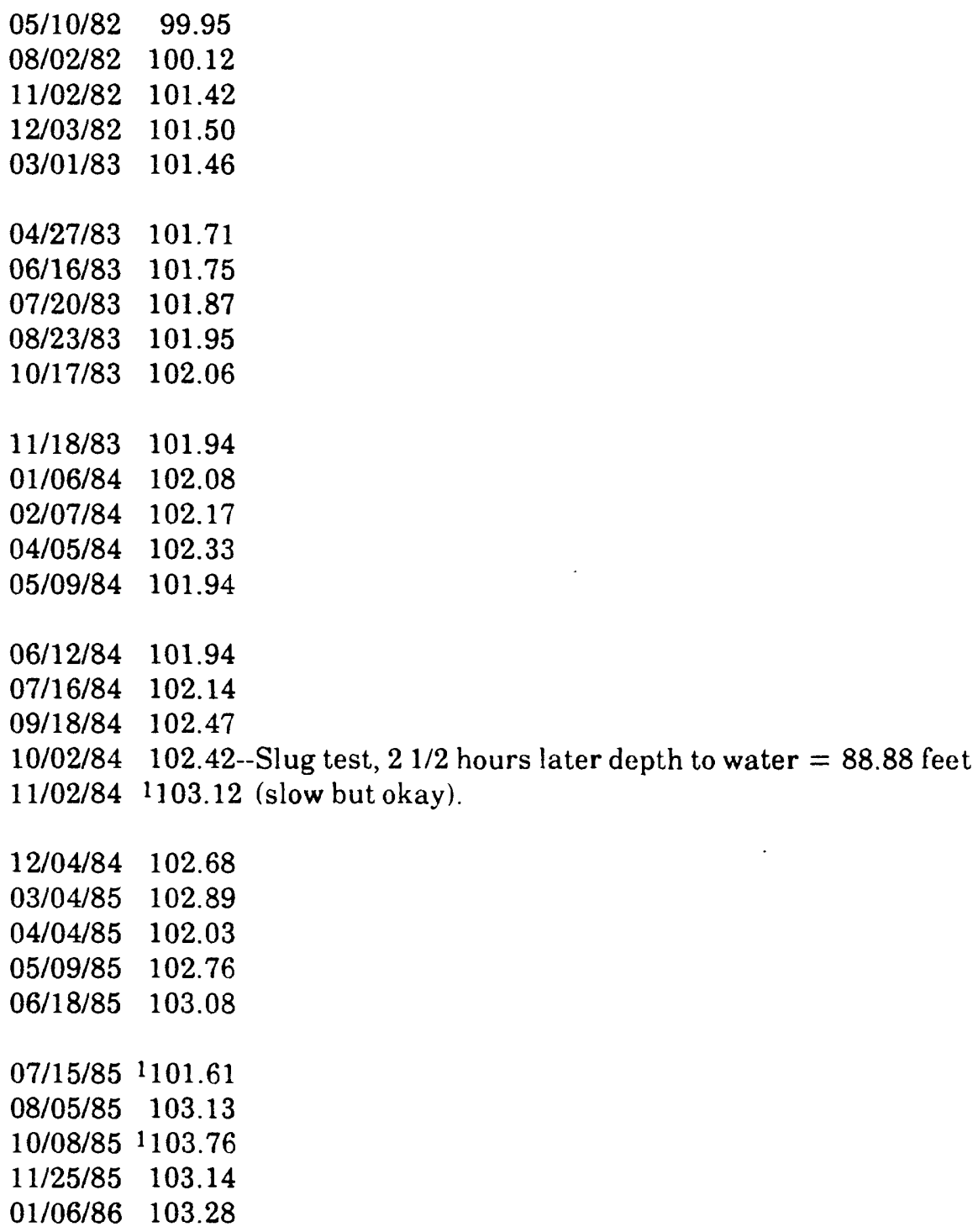


Table 5. Measurement of depths to water in observation wells 1, 2, and 3 made during site visits.--Continued

\begin{tabular}{lcc}
\hline $\begin{array}{c}\text { Date } \\
\text { (month/ } \\
\text { day/ } \\
\text { year) }\end{array}$ & $\begin{array}{c}\text { Depth } \\
\text { to water } \\
\text { (feet) }\end{array}$ & Well 2 \\
\hline $03 / 06 / 86$ & 103.39 & Remarks \\
$04 / 02 / 86$ & 103.42 & \\
$05 / 12 / 86$ & 103.74 & \\
$06 / 23 / 86$ & 103.80 & \\
$07 / 25 / 86$ & 103.90 & \\
& & \\
$08 / 29 / 86$ & 103.89 & \\
$10 / 06 / 86$ & 104.20 & \\
$11 / 13 / 86$ & 104.24
\end{tabular}


Table 5. Measurement of depths to water in observation wells 1,2, and 3 made during site visits --Continued

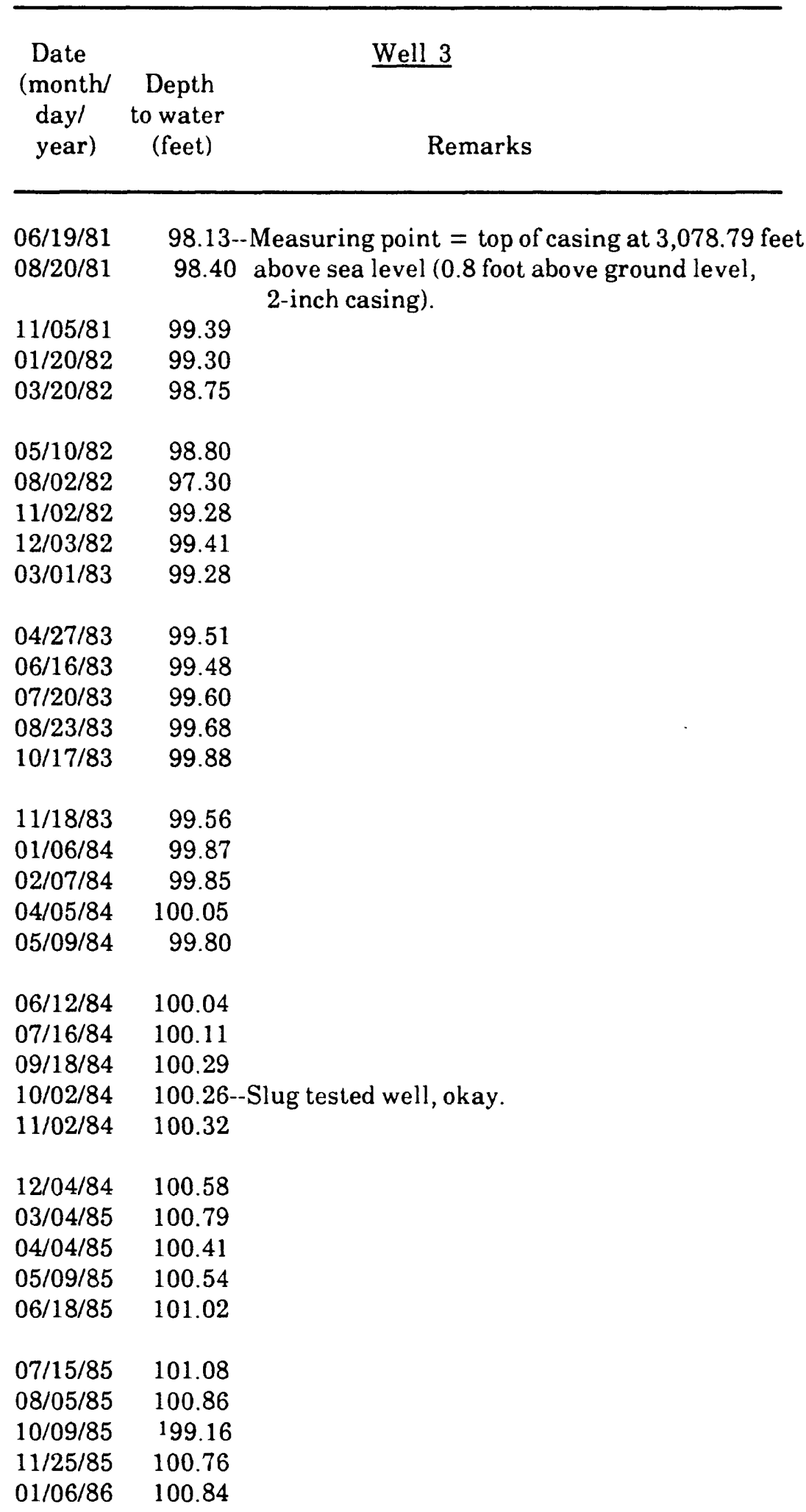


Table 5. Measurement of depths to water in observation wells 1,2, and 3 made during site visits-.-Continued

\begin{tabular}{ccc}
\hline $\begin{array}{c}\text { Date } \\
\text { (month/ } \\
\text { day/ } \\
\text { year) }\end{array}$ & $\begin{array}{c}\text { Depth } \\
\text { to water } \\
\text { (feet) }\end{array}$ & Well 3 \\
\hline $03 / 06 / 86$ & 101.28 \\
$04 / 02 / 86$ & 100.93 & Remarks \\
$05 / 12 / 86$ & 101.49 \\
$06 / 23 / 86$ & 101.60 \\
$07 / 25 / 86$ & 101.62 \\
& & \\
$08 / 29 / 86$ & 101.55 \\
$10 / 06 / 86$ & 101.94 \\
$11 / 13 / 86$ & 101.26 \\
$05 / 12 / 86$ & 94.78 \\
$06 / 23 / 86$ & 94.84 \\
& \\
$07 / 25 / 86$ & 94.87 \\
$08 / 29 / 86$ & 94.88 \\
$10 / 06 / 86$ & 95.24 \\
$11 / 13 / 86$ & 95.11 \\
\hline
\end{tabular}

${ }^{1}$ Measurement noticeably anomalous to others. 


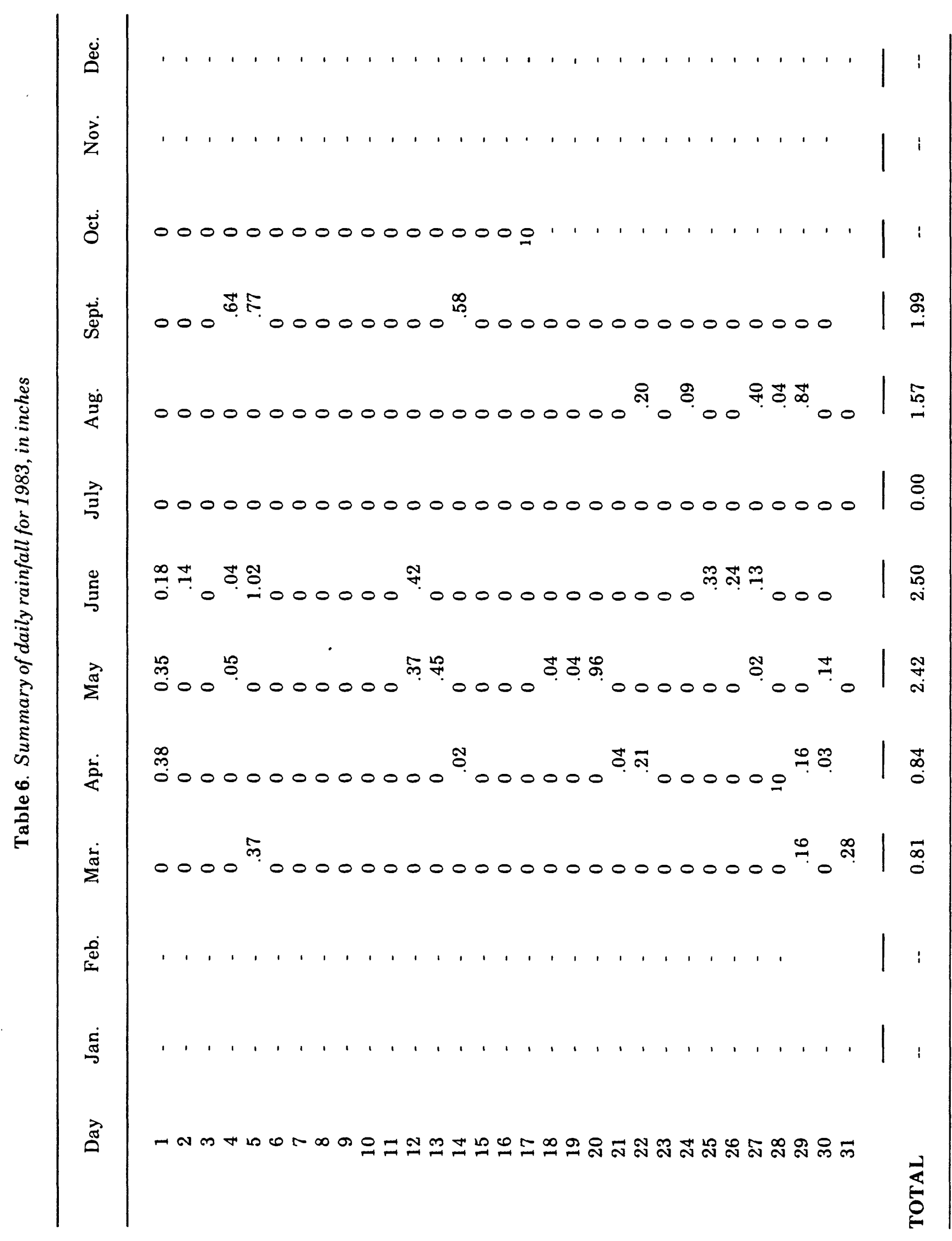




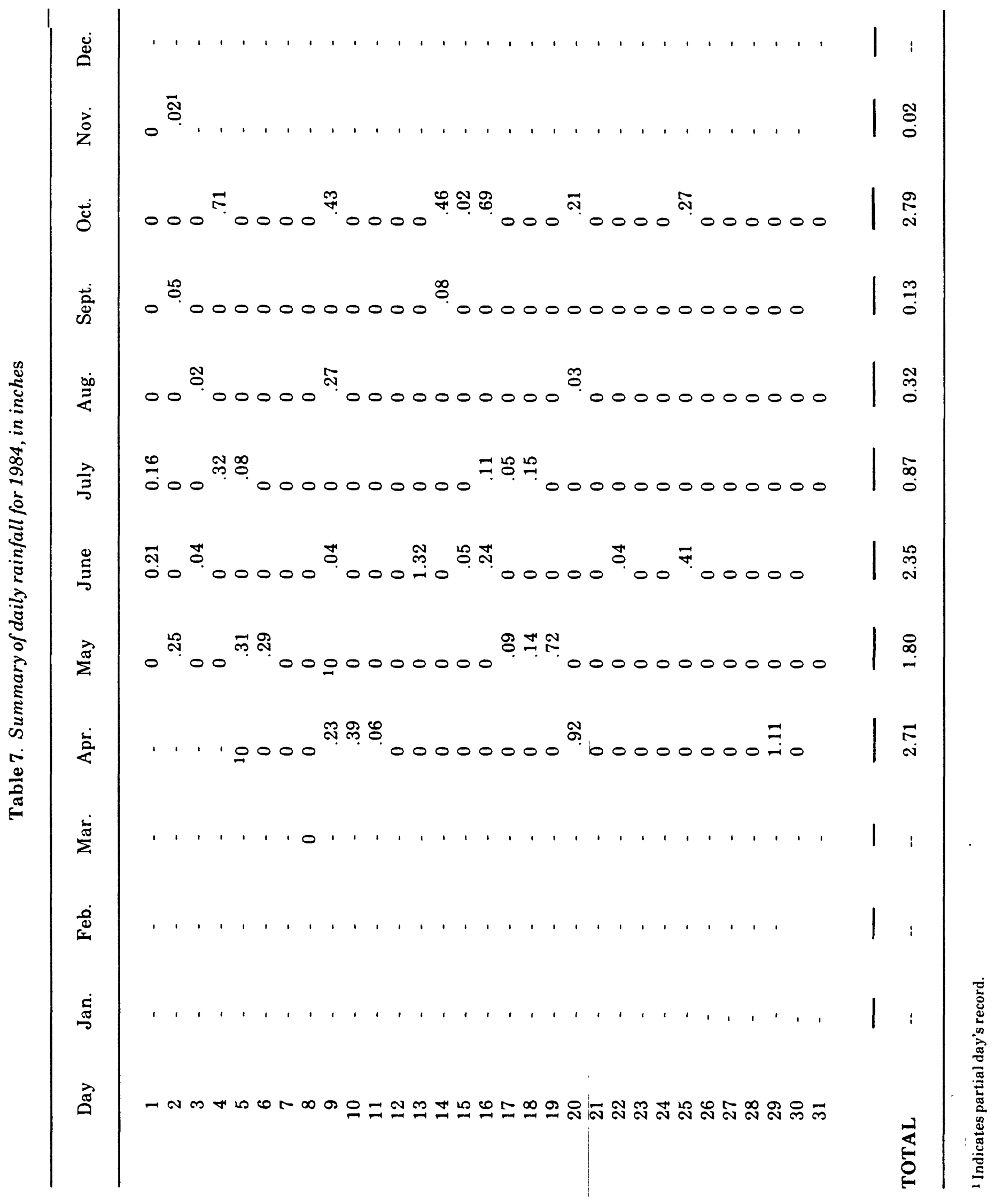




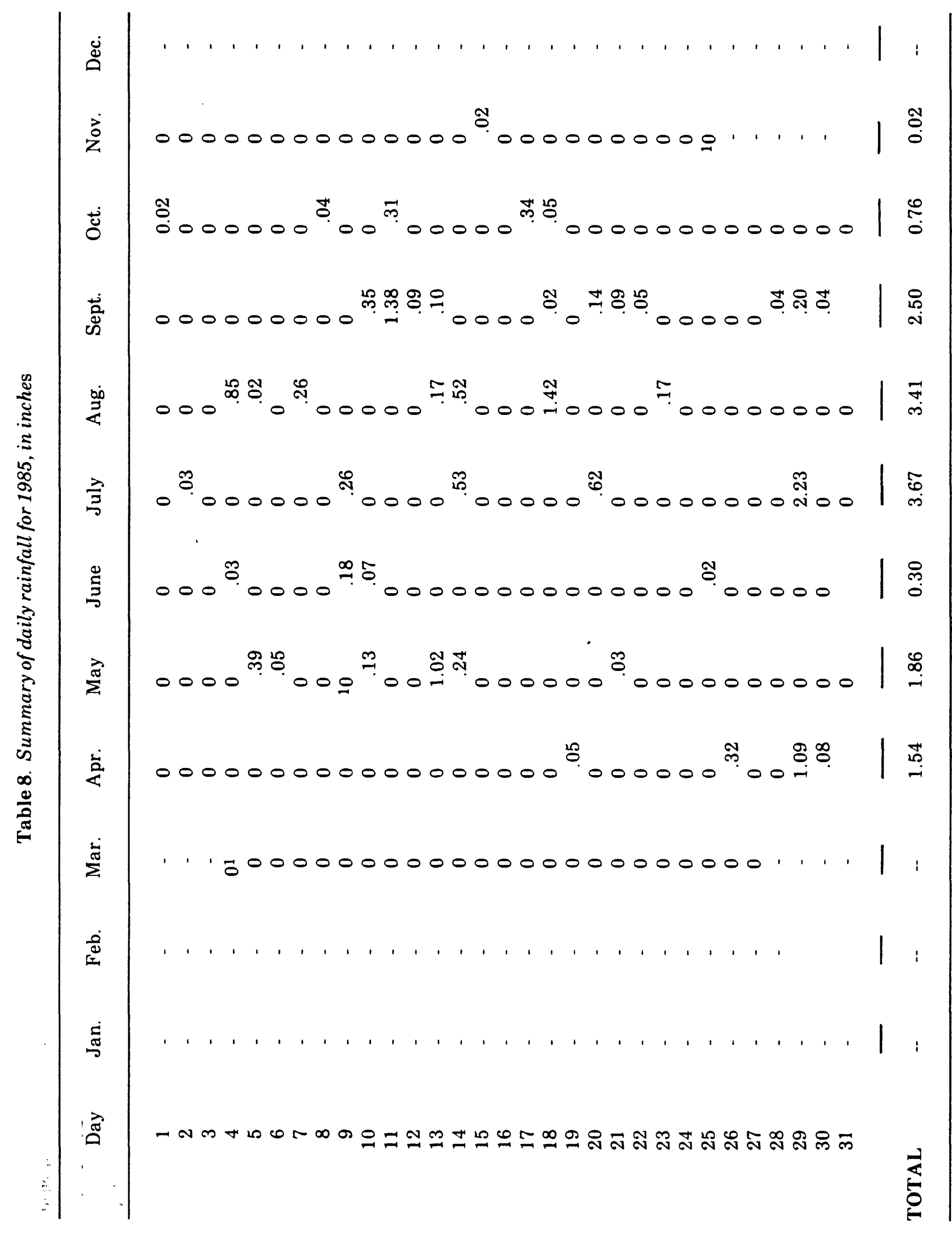




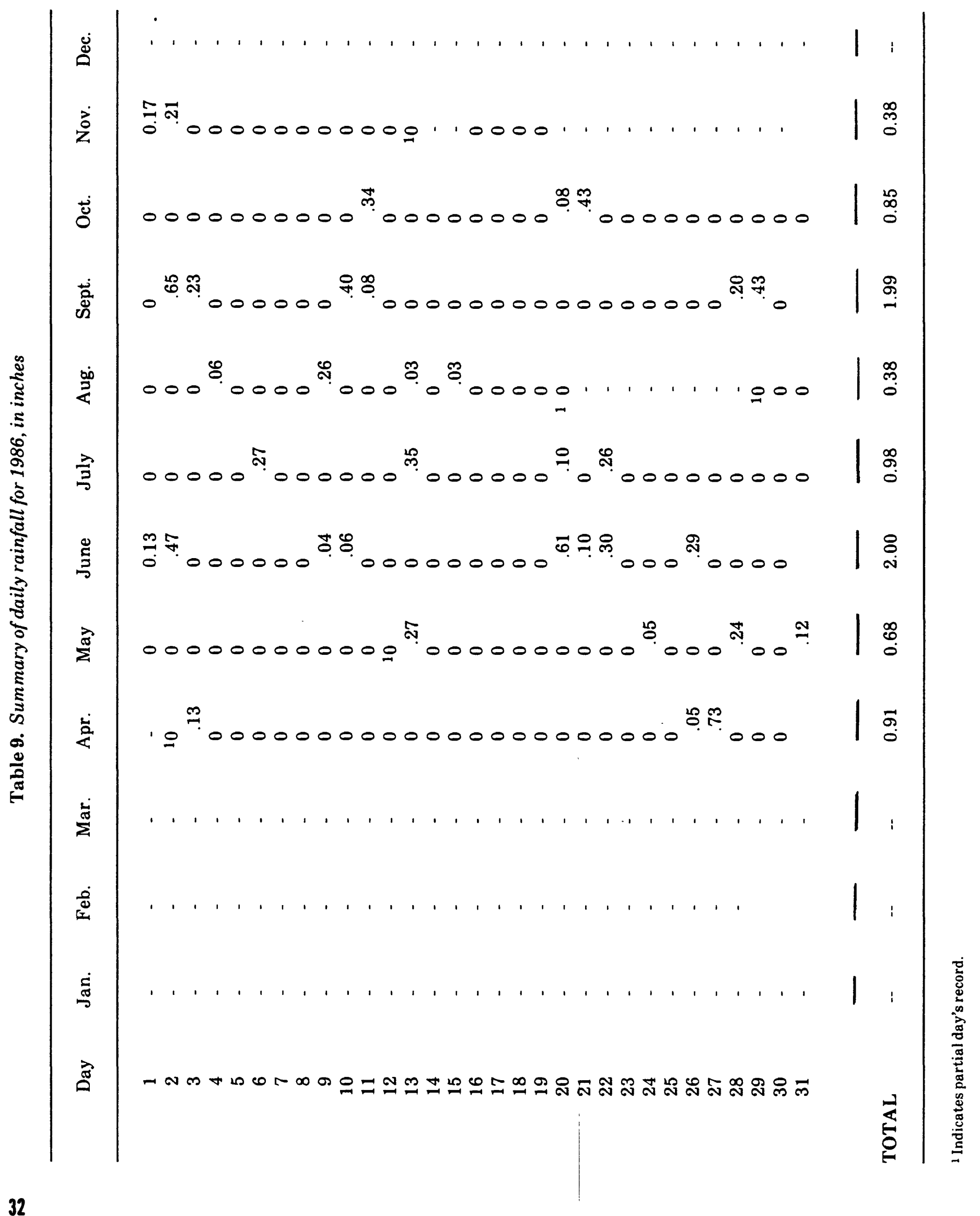


Table 10. Construction data for observation wells

\section{Observation well 1:}

Alias: Hole 705 (construction plans), 17-34W-11BACD.

Drilled: November 8,1979 , with rotary drill, bentonite and revert mud.

Lithologic log (logged by R. H. Drees, U.S. Soil Conservation Service):

Depth, in feet

below land surface

Lithology 1

0.0 to $\quad 0.5 \quad$ Clay, silt, stiff, brown

0.5 to $4.0 \quad$ Silt, sand, light-brown to tan

4.0 to 23.0 Silt, sand, gravel, tan, limey, cemented zone at 19 feet

23.0 to $33.0 \quad$ More silt and clay

33.0 to $\quad 55.0 \quad$ Less silt and clay

55.0 to $88.0 \quad$ Silt, sand, gravel

88.0 to $92.5 \quad$ Caliche, hard to drill

92.5 to $128 \quad$ Soft

128 to $146 \quad$ Clay, silt, sand

Completion data: Hole reamed to 7-7/8 inches down to 146 feet. Placed 143 feet of 4 -inch polyvinyl-chloride pipe in boring (lower 58.3 feet perforated). Annulus gravel packed from bottom to 10 feet, bentonite and clay to surface. Water level, 74.0 feet, November 15, 1979. 


\section{Observation well 2:}

Alias: Hole 704 (construction plans), 17-34W-11BACC.

Drilled: November 7,1979 , with rotary drill, bentonite and revert mud.

Lithologic log (logged by R. H. Drees, U.S. Soil Conservation Service):

Depth, in feet

below land surface

Lithology 1

0.0 to $\quad 0.6 \quad$ Clay, silt, stiff, brown

0.6 to $4.0 \quad$ Silt, clay, light-brown

4.0 to 40.0 Clay, silt, sand, with partially and well-cemented zones (calichelike)

40.0 to $86.0 \quad$ Slightly less "caliche" or hard zones

86.0 to $101.0 \quad$ Hard, very limey (caliche)

101.0 to $135.2 \quad$ Softer

Completion data: Placed 132 feet of 2 -inch polyvinyl-chloride pipe in boring. Annulus gravelpacked from bottom to 5 feet, bentonite and clay to surface. Water level, 88.1 feet, November 8, 1979. Water level, 96.9 feet, November $9,1979$. 
Table 10. Construction data for observation wells--Continued

\section{Observation well 3:}

Alias: Hole 703 (construction plans), 17-34W-11BBAC.

Drilled: November 6, 1979, with rotary drill, bentonite and ruvert mud.

Lithologic log (logged by R. H. Drees, U.S. Soil Conservation Service):

Depth, in feet

below land surface

\section{Lithology 1}

0.0 to $3.0 \quad$ Clay

3.0 to $100.0 \quad$ Clay, silt, sand, gravel, with caliche-like zones.

100.0 to 143.0 More silt and clay, less coarse sand and small gravel.

143.0 to 145.6 Drill action indicates Niobrara Formation, Smoky Hill Member.

Completion data: 143 feet of 2-inch polyvinyl-chloride pipe placed in boring, lower 50 feet slotted with hacksaw, approximately one slot per foot. Annulus gravel-packed from bottom to 5 feet, then bentonite and clay to surface. Water level, 94.0 feet, November 7 , 1979. Water level, 96.3 feet, November 8, 1979. Water level, 96.6 feet, November $9,1979$.

${ }^{1}$ Author's note--Stratigraphic names but no lithology are noted in log. In this report, stratigraphic name is used as descriptor only. 
Refer-

ence Description

mark

RM-1 5/8-inch steel rod at south end of dam, 4 feet from southeast corner post in fenceline around trench silo 300 feet west of section line.

RM-2 Top of steel post near observation well 1 near center of downstream slope of dam.

RM-3 Top of fence post near shelter.

RM-4 3/8-inch bolt set vertically in concrete base of trash guard at north (left upstream) corner, 4 inches out from angle-iron corner post. Normally buried in dirt.

RM-4 End of bolt on interior of lower rail of trash guard at principal spillway at inlet, left alt downstream corner.

Range in gage height, in feet, on staff plates
RP-1 Lag bolt on lowest staff backing.
$0.0-6.7$
RP-2 Lag bolt on staff backing 2 .
$6.8-13.5$
RP-3 Lag bolt on staff backing 3 .
$13.6-20.3$
RP-4 Lag bolt on staff backing 4 (highest)
$23.8-27.1$

MP Top of 4-inch casing, observation well 1--11.2 feet above land surface (May 10, 1982), hole in casing 2.23 feet lower used beginning December 3,1982 , casing cutoff on or about September 29, 1984, to install graphic recorder.

RPW2 Top of post near observation well 2--0.4 foot above ground level.

MP2 Top of 2-inch casing, observation well 2--even with land surface (August 2, 1982).

RPW3 Top of post near observation well 3--0.3 foot above ground level.

MP3 Top of 2-inch casing, observation well 3--0.5 foot above land surface (August 2, 1982).

Nail-in-post is nail set vertically in post at west end of wire gate entrance to site at NE1/4 NE1/4 NW1/4 sec. 11, T. 17 S., R. 34 W.

Ctr-road is center of east-west road at northeast corner of NE1/4 NE1/4 NW 1/4 sec. 11,T. 17 S., R. 34 W. Pence SE U.S. Geological Survey 7.5-minute topographic quadrangle gives that location as 3,103 feet above sea level.

TBM-1 Dam construction mark--5/8-inch steel rod in center of crest of dam, about 90 feet northeast of southwest end of dam. Dam height, 56.20 feet above dam datum. Not found.

TBM-2 Dam construction mark--1/2-inch steel rod in northwest-southeast fenceline 200 feet southeast (downstream) of southwest end of dam. Dam height, 61.8 feet above dam datum. Not found. 
Table 11. Description of reference marks--Continued

Refer-

ence $\quad$.. Description

mark

Crest of inlet--Dam height, 39.1 feet above dam datum (March 20,1987, 16.73 feet gage height).

Crest of emergency spillway--Dam height, 58.0 feet above dam datum (March 20, 1987, 35.8 feet gage height).

Top of dam--Dam height, 62.5 feet dam datum (reportedly built a couple of feet higher by contractor?). 


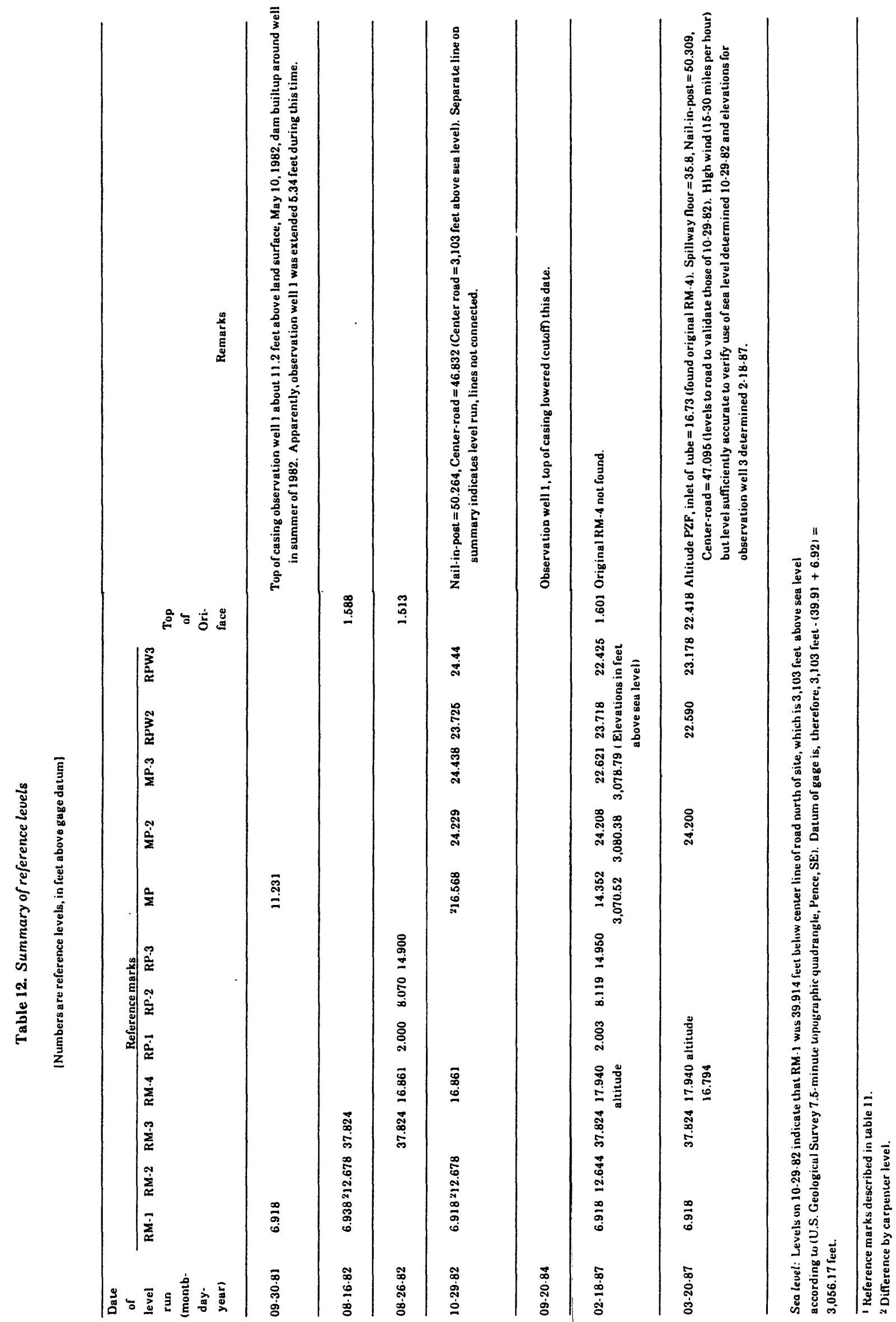

